\title{
Uncertainty in Interval Type-2 Fuzzy Systems
}

\author{
Sadegh Aminifar and Arjuna Marzuki \\ School of Electrical and Electronics Engineering, Universiti Sains Malaysia, Engineering Campus, 14300 Nibong Tebal, \\ Pulau Pinang, Malaysia \\ Correspondence should be addressed to Sadegh Aminifar; sadeghaminifar@gmail.com
}

Received 8 June 2013; Accepted 1 August 2013

Academic Editor: Alexander P. Seyranian

Copyright (C) 2013 S. Aminifar and A. Marzuki. This is an open access article distributed under the Creative Commons Attribution License, which permits unrestricted use, distribution, and reproduction in any medium, provided the original work is properly cited.

\begin{abstract}
This paper studies uncertainty and its effect on system response displacement. The paper also describes how IT2MFs (interval type2 membership functions) differentiate from T1MFs (type-1 membership functions) by adding uncertainty. The effect of uncertainty is modeled clearly by introducing a technique that describes how uncertainty causes membership degree reduction and changing the fuzzy word meanings in fuzzy logic controllers (FLCs). Several criteria are discussed for the measurement of the imbalance rate of internal uncertainty and its effect on system behavior. Uncertainty removal is introduced to observe the effect of uncertainty on the output. The theorem of uncertainty avoidance is presented for describing the role of uncertainty in interval type-2 fuzzy systems (IT2FSs). Another objective of this paper is to derive a novel uncertainty measure for IT2MFs with lower complexity and clearer presentation. Finally, for proving the affectivity of novel interpretation of uncertainty in IT2FSs, several investigations are done.
\end{abstract}

\section{Introduction}

Type-n fuzzy sets were discussed generally and comprehensively in [1-12]. The standardized version of interval type2 (IT2) fuzzy systems (IT2FSs) [13] was referred to in [1416]. The standardized IT2FS was completed in 2007. The IT2FS provides a suitable environment for computing with words (CW) [17]. However, complexities, high volumes of calculations, and ambiguities in applying uncertainty hinder extensive applications. This paper investigates the sources of uncertainty and the uncertainty handling behavior of IT2FSs.

Interval type- 2 fuzzy sets have widely been accepted as more capable of modeling higher orders of uncertainty than type-1 fuzzy sets $[4,9-11,18,19]$. This property has been the driving force behind much of the advancement of interval type-2 fuzzy set theories and applications [10]. One of the most important causes of such forces is finding a suitable interpretation that is theoretically meaningful and practically tractable. Uncertainty plays an important role in fuzzy logic theory and applications. The amount of uncertainty inherent in a fuzzy set has been quantified using different methods. Klir and Parviz presented the generalized information theory as a foundation for a research project that unifies the theories dealing with uncertainty in [20]. In this paper, interval type-2 fuzzy sets (IT2FS) are utilized in the field of generalized information theory and general theory of uncertainty. The contribution presented in this paper paves the way for active research on the relationship between the amount of injected uncertainty and response displacement after injection of uncertainty.

The authors presented an ensemble of fuzzy systems in [21] for capturing uncertainty. In that paper one type1 fuzzy logic controller chooses the output position in an area produced by another type-1 fuzzy system. This paper attempts to discover the new features of IT2FSs, specifically the significant relationship between uncertainty and defuzzification of the center of gravity (COG) to show the effect of uncertainty on system output based on extracted concepts. This study investigates that the output of interval type-2 (IT2) fuzzy logic system (FLS) in comparison with type-1 (T1) FLS moves to points with less uncertainty. Uncertainty can enter into T1FSs and affect system response. The effect of uncertainty is modeled clearly by introducing a technique that describes how uncertainty causes membership degree reduction and changing the fuzzy word meanings. Several criteria are discussed for the measurement of the imbalance rate of internal uncertainty and its effect on system behavior. This paper shows the imbalance rate of uncertainty that can be seen as a criterion for displacing COG. The effect 
of uncertainty on displacing COG is presented as a novel uncertainty measure in this paper.

The concept of uncertainty in fuzzy systems is interpreted in a new manner as illustrated in Figure 1. This research considers the presence of uncertainty injectors and removers different from conventional fuzzy systems (Figure 1). T1 fuzzy systems do not have these two blocks compared with the proposed fuzzy system. The main difference between T1 fuzzy systems and IT2FSs [13] is found in the fuzzifier and defuzzifier blocks.

Uncertainty removal is introduced to observe the effect of uncertainty on the output. In Section 2 (Uncertainty), the conceptual descriptions regarding fuzzification block based on the interpretations derived from this paper are discussed. We explain the application of these concepts in a system wherein each variable corresponds to a number with one interval in each moment. Our method attempts to find a T1 fuzzy membership function for each IT2MF based on the effect of uncertainty on certain data. We also avoid using iterative procedures.

The direct role of uncertainty is introduced as the main difference between T1FSs and T2FSs. The justification of application of IT2FLSs in fuzzy systems is highly dependent on uncertainty issue. This paper aims to recognize different types of uncertainty by introducing new definitions and expressions, thus creating informed and goal-oriented maneuvers during the design process and increasing the application of interval T2 fuzzy controllers.

Another objective of this paper is about new uncertainty measure for interval type-2 fuzzy membership functions (IT2MFs). Uncertainty measure is a necessity because to use fuzzy sets (FSs) as granules in general theory of uncertainty (GTU), which is introduced by Zadeh [22], it is necessary to quantify the uncertainty associated with fuzzy sets (FS) because, based on [23], "once uncertainty (and information) measures become well justified, they can very effectively be utilized for managing uncertainty and associated information. For example, they can be utilized for extrapolating evidence, assessing the strength of relationship between given groups of variables, assessing the influences of given input variables on given output variables, measuring the loss of information when a system is simplified, and the like."

Klir and Folger [24], Klir and Parviz [20], and Harmanec [25] have developed three fundamental principles to guide the use of uncertainty measures under different circumstances.

(1) "The principle of minimum uncertainty, which states that solutions with the least loss of information should be selected, can be used in simplification and conflict resolution problems."

(2) "The principle of maximum uncertainty, which states that a conclusion should maximize the relevant uncertainty within constraints given by the verified premises, is widely used within classical probability framework $[26,27]$."

(3) "The principle of uncertainty invariance, which states that the amount of uncertainty should be preserved in each transformation of uncertainty from one mathematical framework to another, is widely studied in the context of probability-possibility transformation $[28,29]$."

This paper tries to discuss the effect of uncertainty of IT2FS based on the first principal of uncertainty.

The remainder of this paper is organized as follows. Section 2 discusses uncertainty and IT2MF efficacy and presents improvements in this field. Section 3 discusses the imbalance rate of internal uncertainty and its effect on system behavior. Section 4 explains new uncertainty measure. Section 5 formulizes the uncertainty effect on system output. Sections 6 and 7 display the simulation analyses and conclusions, respectively.

\section{Uncertainty}

Zadeh points out in [22] that "uncertainty is an attribute of information" and introduced the general theory of uncertainty (GTU), "because existing approaches to representation of uncertain information are inadequate for dealing with problems in which uncertain information is perceptionbased and is expressed in a natural language." He also states that "In GTU, uncertainty is linked to information through the concept of granular structure, a concept which plays a key role in human interaction with the real world [30-32]."

Fuzzy systems are mainly applied for calculations that use lexical variables (i.e., CW) [33]. Verbal interpretations for different operations are the key elements in CW. For fuzzification, general T2 fuzzy systems are more effective in modeling defined quantities by linguistic constraints. IT2FSs, which have less complexity, have discovered a field of application that is higher than T2 fuzzy sets. This paper focuses on IT2FLSs. Figure 2 shows an example of an IT2MF. The membership degree of $(x=a)$ takes the form of an interval or a line segment $\left[\begin{array}{ll}\mu_{l a} & \mu_{u a}\end{array}\right]$ (Figure 2). The length of this line segment indicates the uncertainty bandwidth $\left(\underline{u}=\mu_{u}\right.$ to $\left.\mu_{l}\right)$ on that point. This paper emphasizes the hypothesis in [34], in which IT2MF serves as the expansion of T1 fuzzy MF with an equal amount of uncertainty expansion at both ends. A principal MF that is located in the middle of the upper and lower bands of uncertainty is defined for each IT2MF, where the membership degree of each member $\mu_{p}$ is the average of the upper and lower membership degrees $\left(\mu_{p}=\left(\mu_{u}+\mu_{L}\right) / 2\right)$ [34]. For each T1MF (which is referred to as "deterministic membership degrees," that is, one-to-one correspondence between each variable and its membership degree) an unlimited number of IT2MFs are created because of the presence of uncertainty on either side of each point of the member function. These IT2MFs are called uncertaintified MFs of the T1MF.

Four sources with different nature were mentioned for uncertainty in [35]. These sources introduce the equal IT2MF for uncertainty modeling. For instance, the uncertainty that is transmitted to the data of a local factor such as noise is completely different from the uncertainty that is an inseparable part of a word in mind. Therefore, the uncertainty should be modeled separately or the description trend of 


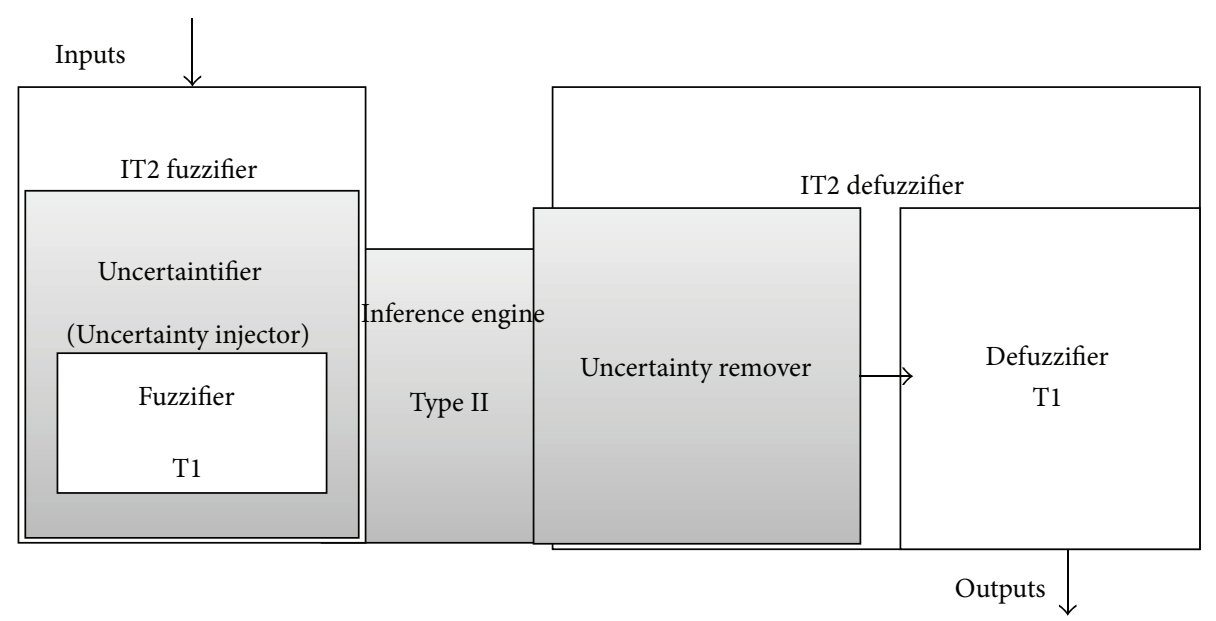

FIGURE 1: The role of uncertainty in an IT2FS or IT2 FLC.

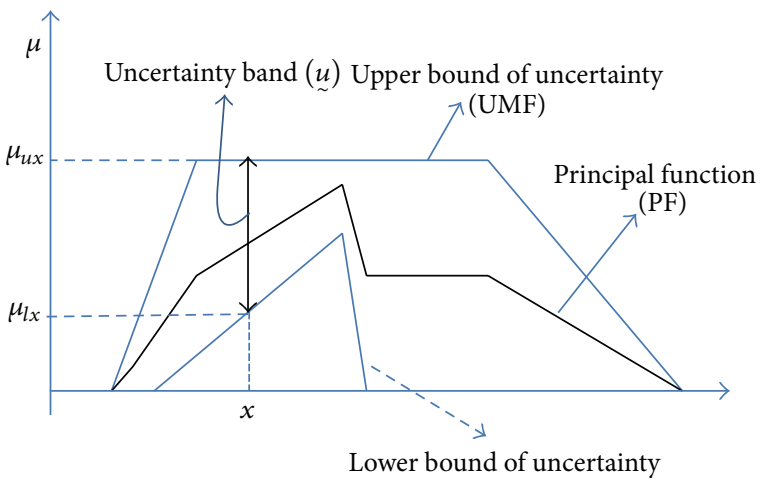

(LMF)

FIGURE 2: Principal function and one of its uncertaintified functions.

this uncertainty should be determined. Several methods of extracting the MF of an IT2FS were explained in [36-38]. Nevertheless, none of these sources have shown clearly the effect of these types of uncertainty on control processes and/or their effect on output. In the literature related to IT2FSs application, the cause of the differences between system outputs in the presence or absence of uncertainty has not been associated with the lack of certainty in a system or data by using documented and significant formulas. Discussions in [39] indicate the minor role of uncertainty in this issue.

If we divide the uncertainty sources introduced in [35] into two groups based on the classifications performed on uncertainty sources [40-42], one group will be related to uncertainty, which naturally exists in every word. This uncertainty is independent from the field wherein adverbs of quantity such as "little," "fairly," "very," and "too" are determined. The quantity and end points of these endpoints have been determined by experts. Mendel proposed a method in [38] to determine the start and end points. Natural uncertainty is nondecreasing; that is, uncertainty may change from person to person and from one period to the next [38], but its nature does not change the general form of the MF.
Modeling the function of interval T2 membership functions (IT2MFs) for this type of uncertainty is very rational. Certain IT2MF words, which are used more in IT2FS, were studied in [18]. These IT2MF words accurately model uncertainty properties within the nature of a word. IT2MF words can be summarized by three modes: small sounding words (low), medium sounding words (medium), and large sounding words (high) (Figure 3) [19]. Gaussian MF is introduced for "high" to generalize the discussion (Figure 3).

In a specific problem, choosing the same type of MF for all inputs and outputs is preferred.

Regardless of whether the triangular, trapezoidal, or Gaussian MFs are selected at points where the input range has quantitatively higher or lower membership degrees, the uncertainty band will be narrower and wider at points that have medium membership degrees, respectively. This model is consistent with our attitudes toward words. For instance, at a low mode, the membership degree is high when the uncertainty of placing very small data in a field becomes low (Points zero and one in low shape in Figure 3). When data becomes larger, the membership degree can be stated more decisively to be not small (Points $c$ and $d$ in low shape in Figure 3). However, at Point $b$, the membership degree cannot be determined at a low mode; furthermore, disagreements among different people and the uncertainty of one person are greater in points close to Point $b$ than the rest of the points (Figure 3).

Another type of uncertainty is related to data. Two out of 4 items discussed in [35] are included in this category. Data uncertainty is not limited to these items, and other items were mentioned in [41, 42]. Most parts of these types of uncertainty can be reduced if a system is better understood. Some types of uncertainty are inevitable because of scientific reasons and the nature of the problem. The efficiencies and abilities of IT2MF have not often been explained clearly. The usual applied forms in [18] and the three basic MFs (Figure 3) against modeling data uncertainty are not as efficient as modeling the uncertainty in the nature of a word. These types of uncertainty are not always in line with the changes of 


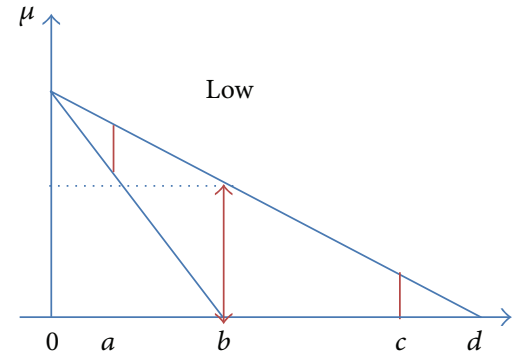

(a)

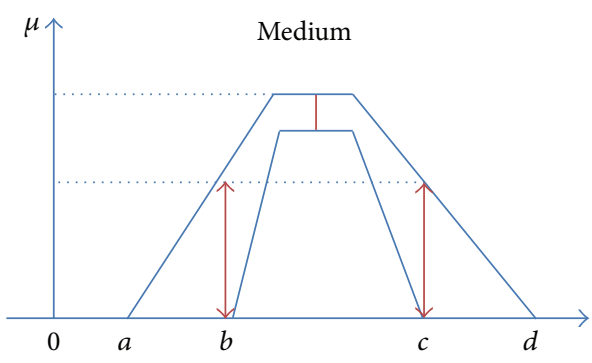

(b)

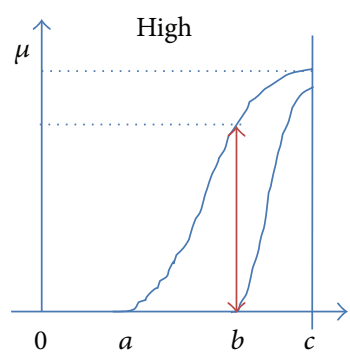

(c)

Figure 3: Basic IT2MF shapes.

TABLE 1: Sample data that denotes uncertainty as an interval.

\begin{tabular}{ccccc}
\hline Interval & {$[2.7,3.3]$} & {$[0.7,3.3]$} & {$[-1.3,3.3]$} & {$[-3.3,3.3]$} \\
Average & 3 & 2 & 1 & 0 \\
\hline Interval & {$[6.3,7.7]$} & {$[5.4,6.6]$} & {$[4.5,5.5]$} & {$[3.6,4.4]$} \\
Average & 7 & 6 & 5 & 4 \\
\hline Interval & {$[11,11]$} & {$[9.825,0.175]$} & {$[8.65,9.23]$} & {$[7.475,8.525]$} \\
Average & 11 & 10 & 9 & 8 \\
\hline
\end{tabular}

the uncertainty band in the nature of a word in terms of decreasing or increasing uncertainty band.

If the uncertainty in the nature of a word is applied to a T1MF, we will achieve the same common IT2MFs. However, if the same approach is implemented on data uncertainty, we will not achieve IT2MFs with common forms. In this example, two types of uncertainties are applied to a basic $\mathrm{T} 1$ fuzzy function in two steps.

Example. Sample data that have been measured for a point obtain an interval for numbers rather than a certain number because of the iteration of measurement or noise. Table 1 shows these intervals and their middle points. The uncertainty bounds of these intervals are the maximum possible bounds in related systems for correspondent data (second row, Table 1). This master interval (the uncertainty of incoming online data does not exceed these intervals) can be achieved clearly from an iterative measurement of a parameter point or from different system conditions, which is applied in a single-input system or a system analyzer. For these recent conditions, the data obtained from two precise temperature sensors can be considered as an interval of uncertainty; the sensors are installed on both ends of an autoclave room or a steam room. The temperature is not necessarily the same at both ends of the room at any moment. The average bounds of the temperature are applied as a single input to the control system of the steam room temperature. This type of uncertainty is created to remove the complex modeling of temperature changes inside an autoclave room.

In this example, we assume that the general field of the data related is the verbal expression of "low," which is defined as T1 fuzzy MF (Figure 4(b)). If the low bound of all points in the intervals of the first row of Table 1 (also shown in Figure 4(a)) is determined by the verbal expression "low" with the MF shown in Figure 4(b) (according to the graphical description of Figure 4(b)), an IT2MF will be created. After deleting the saturated part, an IT2MF is achieved (Figure 4(c)); that is, the IT2MF is obtained from direct inclusion of the data uncertainty in Table 1 to the T1MF of Figure 4(b).

Each set of optional data, which represents one set of figures in each interval of uncertainty in Table 1, has a firstdegree fuzzy MF embedded in the IT2MF of Figure 4(c).

In the following step, we intend to inject data uncertainty to an IT2MF that defines only the natural uncertainty inside the verbal expression (Figure 5(a)). We repeat all the abovementioned steps for every single T1 embedded membership degree function in Figure 4(c), which is limited to the boundaries shown in Figure 5(b) and takes the form of Figure 5(c).

According to the definition provided in this section, the MFs of Figures 4(c) and 5(c) are the IT2MFs for "low" (also called uncertaintified) of Figure 4(b) per data uncertainty and per natural uncertainty, respectively.

The effect of these different MFs on system behavior is investigated in Section 6 (Investigation 6).

\section{Rate of Internal Uncertainty Imbalance and Its Effect on System Response}

Discussions have been provided in [18] regarding uncertainty measurement. By considering the concepts of these discussions, new definitions that are compatible with the objectives of this paper are presented in this section. The difference in the internal uncertainty of words plays an important role in finding the COG through the principles introduced in the following sections of the present paper. Thus, a new formula and definition is provided for COG calculation. Before starting the discussion, we define the function of the absolute ratio of two parameters:

$$
A R(A, B)=\left(\left(\frac{A}{B} \text { if } B<A\right),\left(\frac{B}{A} \text { if } B<A\right)\right) .
$$

Definition 1. For each IT2MF, one defines a function of $\underset{\sim}{u}$, which shows the bandwidth of uncertainty $\left(\underline{u}=\mu_{u}-\mu_{l}\right)$ for each point of horizontal axes of IT2MF as shown in Figure 6. 


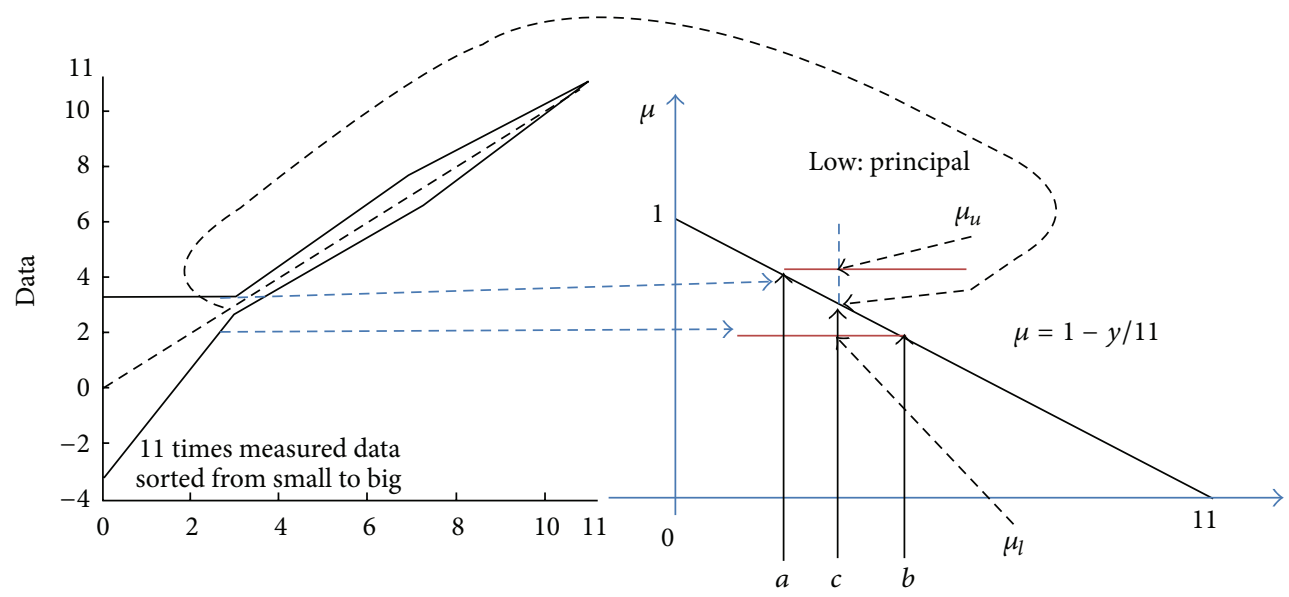

(a)

(b)

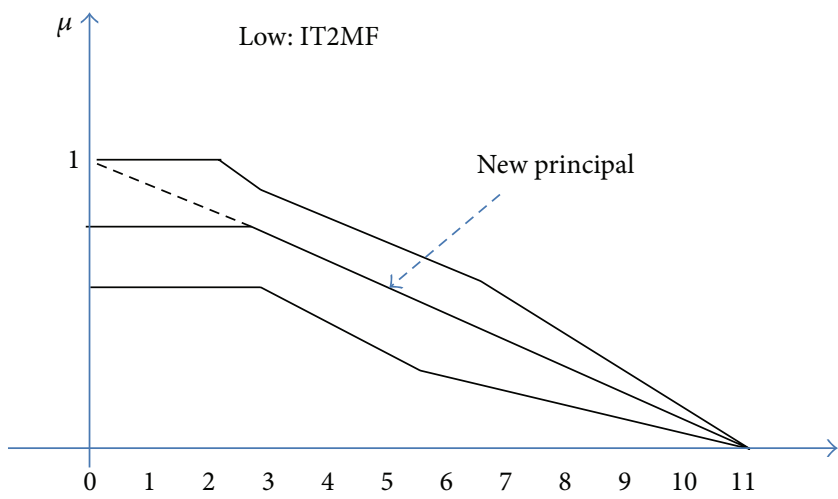

(c)

FIGURE 4: (a) Uncertain space of measured data. (b) Application of uncertain space to one TIMF. (c) Obtained IT2MF.

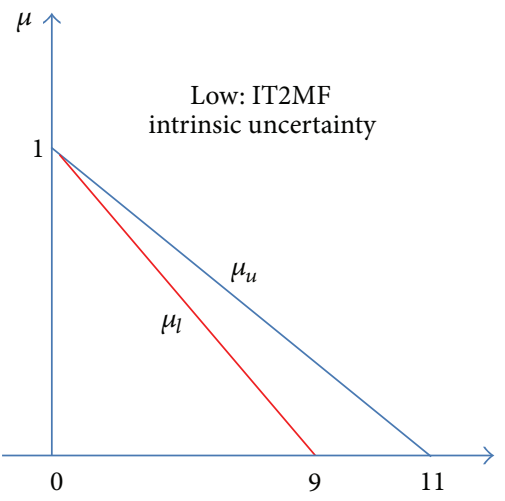

(a)

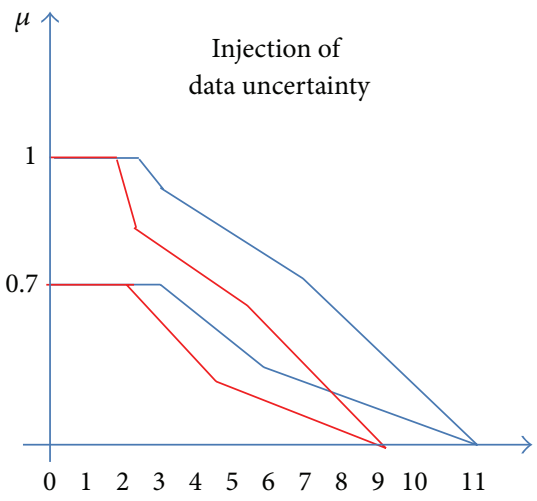

(b)

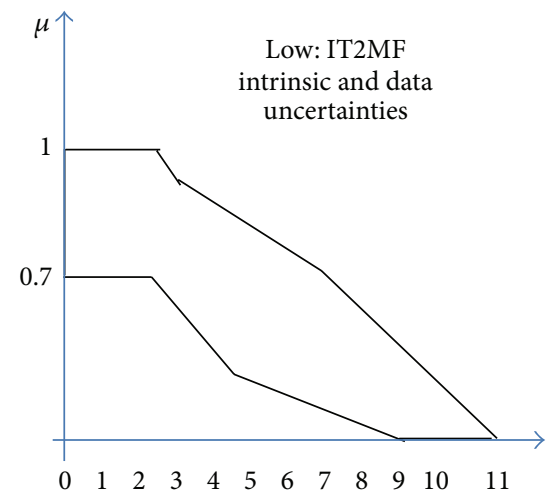

(c)

FIGURE 5: (a) IT2MF based on the intrinsic description of uncertainty. (b) Application of uncertain space to one type-2 MF. (c) Obtained IT2MF.

Definition 2. The absolute ratio of the surface areas on both sides of a line to the length of the COG of the principle function enclosed between the upper and lower bounds of uncertainty band is called the difference index in internal uncertainty, also referred to as the absolute ratio of surface (ARS). If the uncertainty bandwidth by $\underline{u}$ and $\underline{u}(y)$ is a function that shows the uncertainty bandwidth of every single point of the IT2MF of $F$, the amount of ARS is shown as follows:

$$
\operatorname{ARS}(F)=A R\left(\int_{a}^{M} \underline{U} d y, \int_{M}^{b} \underline{U} d y\right) .
$$

The role of ARS, which is the role of the surface area between two uncertainty bands in internal uncertainty in 


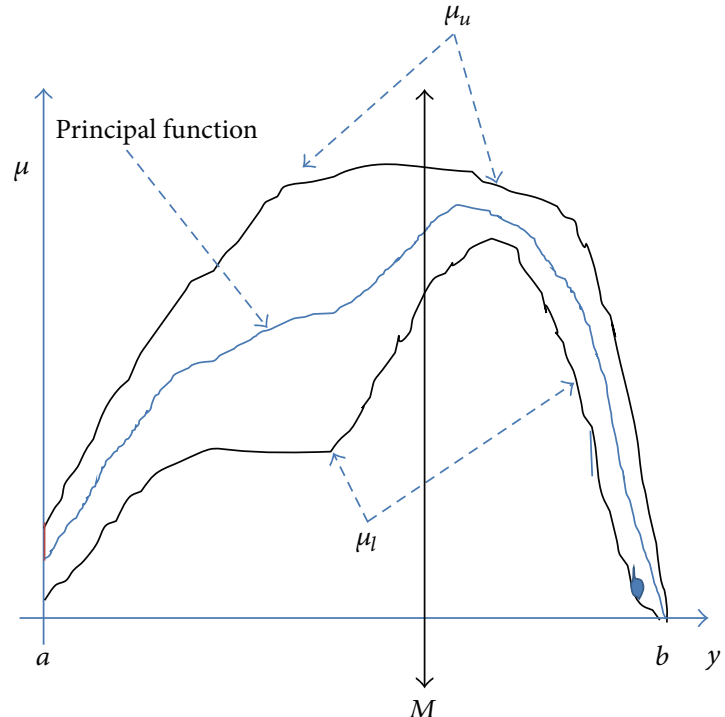

(a)

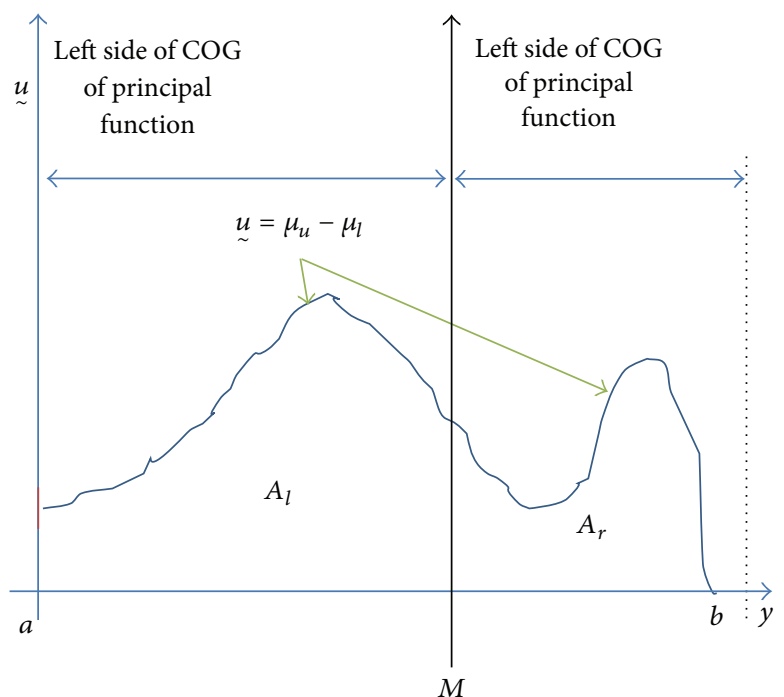

(b)

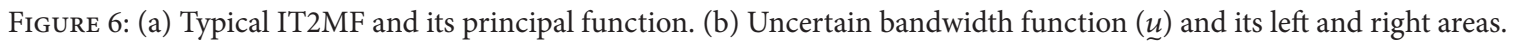

[18], is used in the remaining sections of this paper to prove the theorem proposed in this section. ARS is also used as a closed formula for finding COG as a factor to drift the COG.

Theorem of Uncertainty Avoidance. In proportion to the COG of the principal MF, the COGs of uncertaintified MFs are displaced toward a more clarified domain. A higher amount of uncertainty imbalance on both sides of the COG of the principle MF corresponds to more displacements that shift toward certainty.

For example, as shown in Figure 7, if some uncertainty is injected to one side of the COG of IT2MF, the COG will shift toward the other side. An increasing uncertainty on the left of Points $b$ and $c$ displaces the response (COG) toward the right compared with Point a (Figure 7). In Points $c$ and $d$, creating a slight uncertainty toward the right slightly displaces the response (COG) toward the left. The blue shadow around each point shows the uncertainty around that point (Figure 7).

Verification. We use the physical properties of the COG of homogeneous planes without employing large volumes of calculations, even simple ones, to prove the theorem. An IT2MF is formed by creating uncertainty at both sides of the function that is on the left side of the COG (Figure 8(b)). The MF of Figure 8(a) is the principal function of this T2MF. According to the principles of the Karnik-Mendel (KM) calculations [34], the switching $L$ and $R$ points are located on the left and right sides of $M$, respectively; $L$ and $R$ are the COGs of T1 embedded fuzzy MF, respectively (Figures $8(\mathrm{c})$ and $8(\mathrm{~d}))$. By contrast, in the homogeneous plane below the curve of the principal function, the $s_{1}$ and $s_{2}$ domains are reduced (cut) from the left side of their COG (Figure 8(d)). This makes the new COG (i.e., $R$ ) move toward the right side

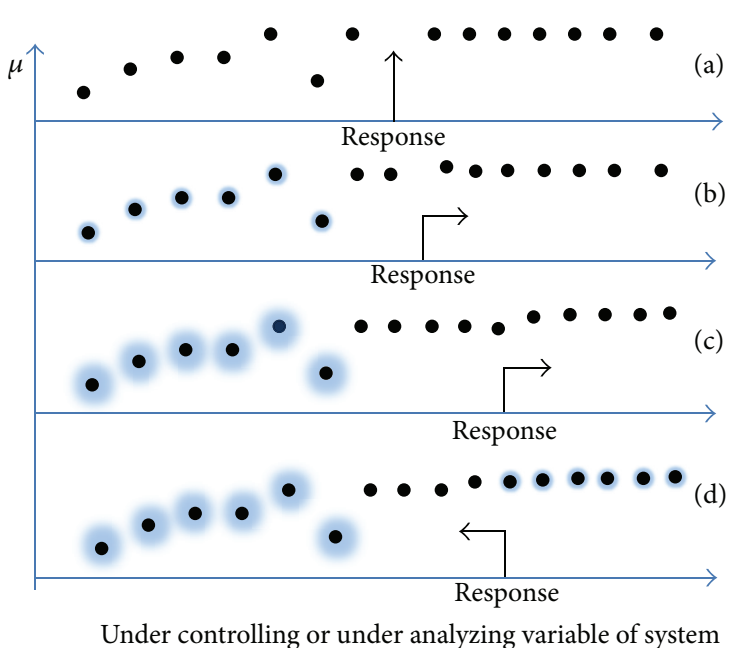

FIgURE 7: (a) Both sides are certain; (b) left side is slightly uncertaintified; (c) left side is more uncertaintified; (d) addition of uncertainty in the right side of (c).

of $M$ as far as $d_{r}$. The addition of the $s_{1}$ domain makes the COG move left, and the $s_{2}$ domain is removed because of the movement of the COG to the right (Figure 8(c)). The effect of the $s_{1}$ and $s_{2}$ domains will not cause COG to exit toward the right side of $M$. The uncertainty grows equally at both sides of the principal function. The $s_{1}$ and $s_{2}$ domains in both Figures $8(\mathrm{c})$ and $8(\mathrm{~d})$ have similar effects on the movement of COG (in the same and opposite directions).

It is concluded that $d_{l}<d_{r}$, considering that the effect of both domains on COG displacement is in the same direction in Figure 8(c) and in opposite direction in Figure 8(d). Therefore, the center of gravity of IT2MF function moves toward the right side of $M$, as shown in Figure 8(b). 


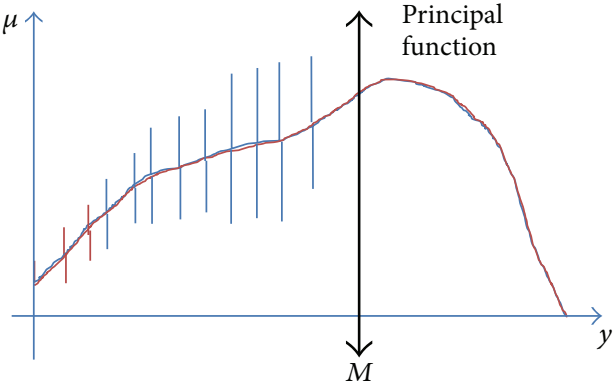

(a)

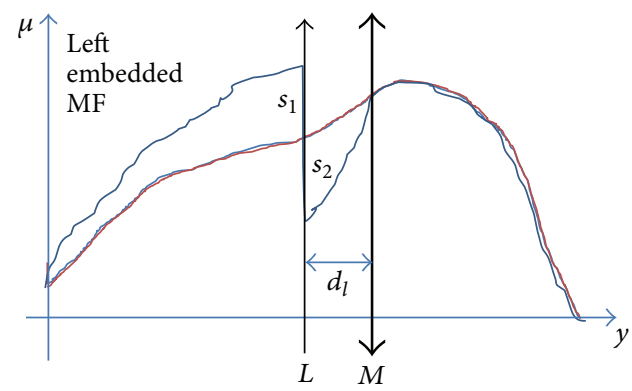

(c)

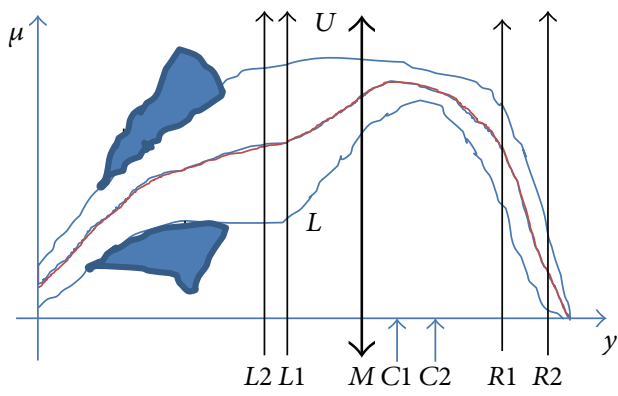

(e)

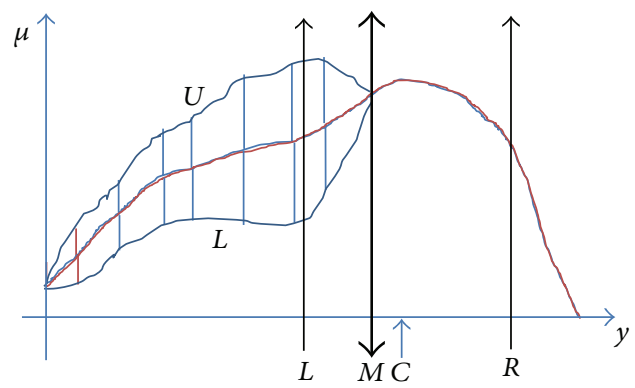

(b)

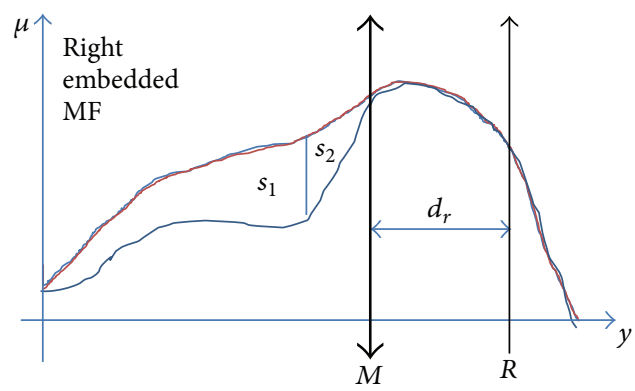

(d)

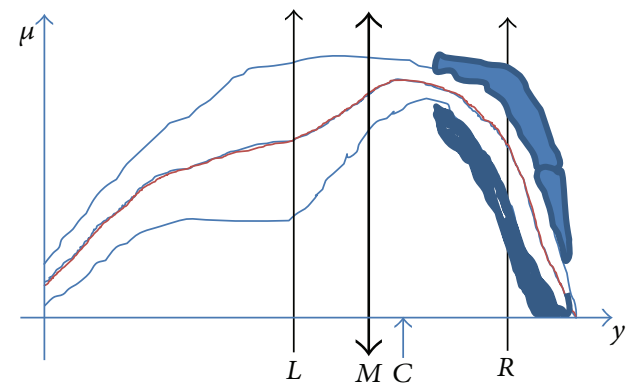

(f)

FIGURE 8: IT2MF that indicates the upper bound; $L 1$ is the lower bound; $m$ is the principal function; $M$ is the COG of $m$; $C$ is the COG of IT2MF.

Figure 8(e) shows an IT2MF with the same principal function as the IT2MF of Figure 8(a). The COG and switching points of this IT2MF are $C 1, L 1$, and $R 1$. A solid color domain is added, thus, increasing uncertainty. Given that the upper domain is considered the domain added to the left side of $L 1$ and T1 fuzzy function, in which $L 1$ is the COG, COG departs from $M$ toward the left. In addition, the lower solid domain acts as the domain reduced from the T1 fuzzy function, in which $R 1$ is the COG. This setting causes the COG to depart from $M$ toward the right side. The movements of the right and left COGs do not help displace $C$. Two solid domains are not added to or reduced from an identical function; however, one domain was added to $U$ and the other was reduced from $L$. Considering that $L$ is always smaller than $U(L<U), d_{r}$ is bigger than $d_{l}\left(d_{r}>d_{l}\right)$; thus, C2 $>C 1$.

The amount of uncertainty added to the right and left sides of $R$, which is proven by the previous procedure, moves the COG toward the left side.

\section{Proposed Uncertainty Measurement Method}

Theorem of Uncertainty Avoidance points out that "The response of system avoids uncertainty." In case of IT2FMs, it means that the center of gravity of uncertaintified membership functions is displaced toward the less uncertaintified domain or, in other words, toward the more clarified domain. The more the amount of imbalance of the created (or available) uncertainty on both sides of the center of gravity of principle membership function, the more the displacement will tend toward certainty. In other words, if some uncertainty is injected to one side of the center of gravity of IT2MF, the center of gravity will be shifted toward the other side.

In this paper, we measure the rate of uncertainty based on the power of total uncertainty to push the response of system to the opposite side considering the aforementioned theorem.

In this method, we add a completely certain membership function (with membership degree is equal to one) to IT2MF in the right hand in such a way that the COG 


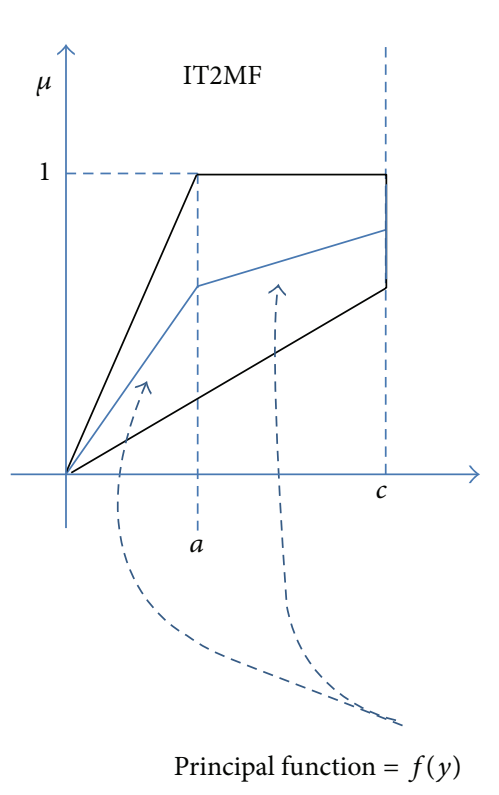

(a)

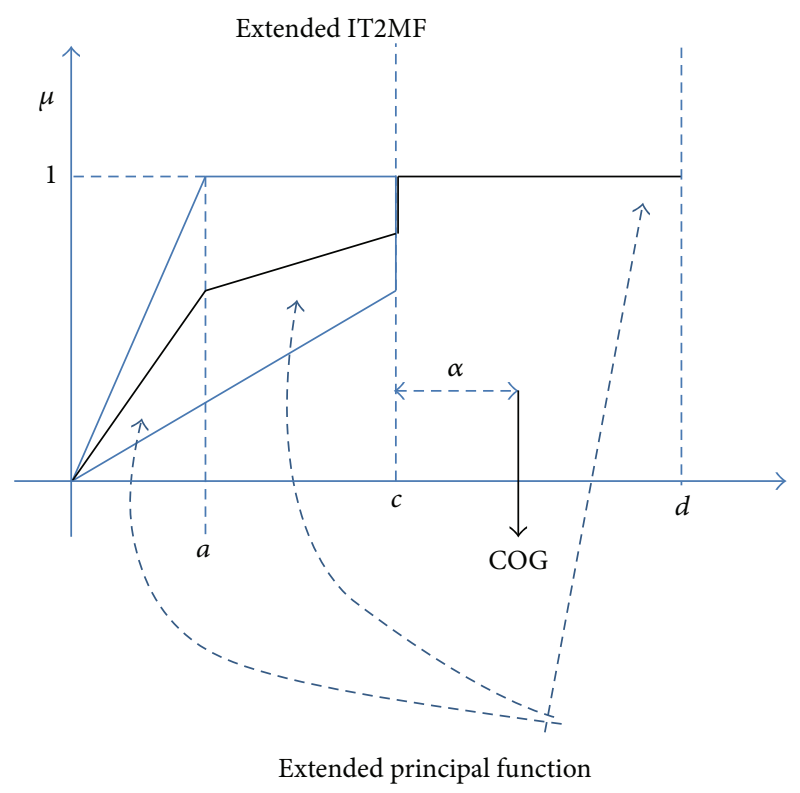

(b)

FIgure 9: (a) A typical IT2MF. (b) Extended principal function and extended IT2MF.

of new established principal function is positioning in the conjunction point of the added part with earlier IT2MF.

Considering the aforementioned theorem, the COG of new established IT2MF must be displaced to the right side in (Figure 9(b)), which is completely certain.

Method 1. In Method 1, we use KM algorithm for calculating COG of IT2MF. The procedure of calculation has been described below in detail.

(1) The domain of discourse of shown IT2MF in Figure 9(a) is from zero to $y=c$. The principal function also has been shown in Figure 9(a).

(2) The principal function must be extended to the right side by adding a $\mathrm{T} 1$ membership function with fixed and certain membership degree of "one." We lengthen the added part to the right in Figure 9(b) so that the COG of total new established principal function is positioning in $y=c$.

(3) For obtaining " $d$ ", (3) is written to show that $y=c$ is the COG of new principal function. From this equation the second-degree equation (4) is concluded, in which just " $d$ " is unknown:

$$
\begin{gathered}
c=\frac{\int_{0}^{c} f(y) y d y+\int_{c}^{d} 1 y d y}{\int_{0}^{c} f(y) d y+\int_{c}^{d} 1 d y} \\
=\frac{\int_{0}^{c} f(y) y d y+(1 / 2)\left(d^{2}-c^{2}\right)}{\int_{0}^{c} f(y) d y+(d-c)} \\
\frac{1}{2} d^{2}-c d+\left(\int_{0}^{c} f(y) y d y-\int_{0}^{c} f(y) d y-\frac{1}{2} c^{2}+c\right)=0 .
\end{gathered}
$$

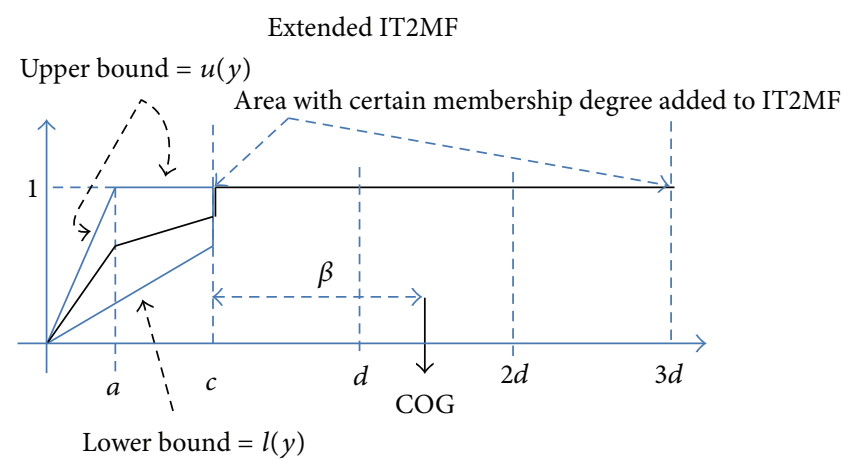

FIGURE 10: Extended IT2MF based on Method 2.

(4) Using KM algorithm, we calculate the COG of extended IT2MF.

(5) The direct distance between $y=c$ and $y=$ (new IT2MF COG), which is shown with $\alpha$ in Figure 9(b), is a criterion of pushing power of the IT2MF uncertainty. We can compare the uncertainty inside of a set of IT2MFs, which are put in a domain by calculating $\alpha$.

Method 2. In this method, we use an approximation for calculating the COG of IT2MF during extracting a criterion for measuring uncertainty.

In this method, we extend the IT2MF as described in part 2 of Method 1 but not with the same length of principal function. In this method, " $d$ " is multiplied by " $n$ " as shown in Figure 10. Enlarging " $d$ " to $n$ times helps us to use $\mathrm{KM}$ algorithm in closed form formula with acceptable approximation for calculating COG of extended IT2MF. For larger $n$, the error caused by this approximation is negligible. 
It should be considered that higher $n$ decreases the sensitivity of $\beta$ uncertainty measure criteria.

(1) Given that switching point in KM algorithm is positioned in the right side of $y=c y_{\text {right KM }}$ is easily obtainable from

$$
\begin{aligned}
y_{\text {right KM }} & =\frac{\int_{0}^{c} u(y) y d y+\int_{c}^{n d} 1 y d y}{\int_{0}^{c} u(y) d y+\int_{c}^{n d} 1 d y} \\
& =\frac{\int_{0}^{c} u(y) y d y+(1 / 2)\left((n d)^{2}-c^{2}\right)}{\int_{0}^{c} u(y) d y+(n d-c)} .
\end{aligned}
$$

(2) Given that switching point in KM algorithm is positioned in the right side of $y=c y_{\text {left KM }}$ is easily obtainable from

$$
\begin{aligned}
y_{\text {left KM }} & =\frac{\int_{0}^{c} l(y) y d y+\int_{c}^{n d} 1 y d y}{\int_{0}^{c} l(y) d y+\int_{c}^{n d} 1 d y} \\
& =\frac{\int_{0}^{c} l(y) y d y+(1 / 2)\left((n d)^{2}-c^{2}\right)}{\int_{0}^{c} l(y) d y+(n d-c)} .
\end{aligned}
$$

(3) The COG of extended principal function is shown in

$$
y_{\mathrm{COG}}=\frac{y_{\text {right KM}}+y_{\text {left KM }}}{2} .
$$

(4) $\beta$ is introduced as a criterion for uncertainty measure by

$$
\begin{aligned}
\beta=y_{\mathrm{COG}}-c= & \frac{\int_{0}^{c} u(y) y d y+(1 / 2)\left((n d)^{2}-c^{2}\right)}{2 \int_{0}^{c} u(y) d y+2(n d-c)} \\
& +\frac{\int_{0}^{c} l(y) y d y+(1 / 2)\left((n d)^{2}-c^{2}\right)}{2 \int_{0}^{c} l(y) d y+2(n d-c)}-c .
\end{aligned}
$$

\section{Uncertainty Effect on Output}

According to Theorem of Uncertainty Avoidance, if uncertainty increases in one side of COG of principal function, the new COG moves to the other side. In other words, we can see this effect similar to the situation in which the membership grades of principal function are decreased in that side that uncertainty increased. Equation (9) is introduced to describe the behavior of this imbalanced internal uncertainty, based on Theorem of Uncertainty Avoidance and first principal of uncertainty of Klir (refer to Section 2). This formula shows that new membership degrees decrease after uncertainty removal. The COG of $\mu_{d}$ is compatible with the COG of an IT2MF:

$$
\mu_{d t r}=\mu_{p}-\frac{1}{2+s_{1}} \underline{u}^{1+s_{2}} .
$$

$s_{1} \geq 0$ and $s_{2} \geq 0$ are parameters that depend on various IT2MF factors. The overall formula, which can be deducted from this paper, for the uncertainty effect on the MF and membership degree should satisfy the following conditions. Theorem of Uncertainty Avoidance should be considered in the overall formula because the resulted property is the main reason to differentiate T1 and T2 fuzzy systems. Another condition is the decrease of membership degrees. The COG is displaced by data that are more deterministic with respect to the difference of uncertainty at both sides of the COG of the principal function. In the last part, attempts are made to introduce the special formula, whose output shows the effect of uncertainty. To reduce the error of the formula under special conditions, wherein we insist the output is COG, the formula is improved as in the following method. The following formula was introduced according to two conditions mentioned previously and produces a proper output. This method has free parameters that can be determined by a designer or the nature of the problem to obtain favorable results such as the COG.

Considering the definition of ARS in (2), ARS is always between zero and one. With respect to the performed simulations, the increase in the distance of ARS from zero and one corresponds to higher errors. Thus, the sensitivity of COG in medium ARS severely devaluates unreliable data. Based on this fact, the behavior of the COG of IT2MF (i.e., (9)) is specialized into (10). Equation (10) approximately produces the COG of IT2MF:

$$
\mu_{d t r}=\mu_{p}-\frac{1}{2+(1 / \mathrm{ARS}) * \mu_{p}} \underset{\sim}{\mathcal{u}^{2}} .
$$

In the general condition of the proposed method, the response of IT2FS is not always completely in accord with COG. We propose a technique that considers this difference (refer to Table 2) in real problems for designers that want to use the proposed method.

The following results are obtained from the many calculations and simulations conducted by the authors of this paper to extract the closed formula of COG and propose simple and effective formulas.

According to the aforementioned equations, $\mu_{d t r}$ depends on the power of IT2MF. No precise criterion exists to calculate IT2MF under these conditions. The $s_{1}$ and $s_{2}$ domains in (9) are not exactly equal to power but are the criteria of power. $S$ is a better describer for showing the power of IT2MF, the rate of absence of uncertainty, and the presence of higher membership degrees. The answer will also be nearer to the COG of IT2MF.

Calculations and simulations at various different conditions lead to more complex results. The COG can be shifted by the proposed formula and by calculating the COG by using the $\mathrm{KM}$ algorithm. However, one approach is slower or faster than the other approach.

According to the results obtained from the simulation and calculation in which IT2MF is more asymmetric but has equal uncertainty areas in both sides of the COG of the principal function, (10) acts worse. When ARS distances from zero and one, the behavior of the COG detector is intensified to weaken ambiguous data. 
TABLE 2: Basic IT2FS words: defuzzified output obtained by using the proposed method and difference percentage compared to the COG calculated by the KM algorithm.

\begin{tabular}{|c|c|c|c|c|c|c|}
\hline & UMF & LMF & KM & Proposed & ARS & $\% \mathrm{E}$ \\
\hline$\overline{(1)}$ & {$[0,0,0.14,1.97,1]$} & {$[0,0,0.05,0.66,1]$} & 0.47 & 0.49 & 0.27 & 1.0 \\
\hline$(2)$ & {$[0,0,0.14,1.97,1]$} & {$[0,0,0.01,0.13,1]$} & 0.56 & 0.61 & 0.96 & 2.5 \\
\hline (3) & {$[0,0,0.26,2.63,1]$} & {$[0,0,0.05,0.63,1]$} & 0.63 & 0.66 & 0.47 & 1.1 \\
\hline$(4)$ & {$[0,0,0.36,2.63,1]$} & {$[0,0,0.05,0.63,1]$} & 0.64 & 0.67 & 0.49 & 1.1 \\
\hline$(5)$ & {$[0,0,0.64,2.47,1]$} & {$[0,0,0.10,1.16,1]$} & 0.66 & 0.66 & 0.24 & 0.0 \\
\hline$(6)$ & {$[0,0,0.64,2.63,1]$} & {$[0,0,0.09,0.99,1]$} & 0.67 & 0.68 & 0.29 & 0.3 \\
\hline$(7)$ & {$[0.59,1.50,2.00,3.41,1]$} & {$[0.79,1.68,1.68,2.21,0.74]$} & 1.75 & 1.74 & 0.45 & 0.3 \\
\hline$(8)$ & {$[0.38,1.50,2.50,4.62,1]$} & {$[1.09,1.83,1.83,2.21,0.53]$} & 2.13 & 2.11 & 0.75 & 0.5 \\
\hline$(9)$ & {$[0.09,1.25,2.50,4.62,1]$} & {$[1.67,1.92,1.92,2.21,0.30]$} & 2.19 & 2.28 & 0.99 & 0.2 \\
\hline$(10)$ & {$[0.09,1.50,3.00,4.62,1]$} & {$[1.79,2.28,2.28,2.81,0.40]$} & 2.32 & 2.33 & 0.91 & 0.2 \\
\hline$(11)$ & {$[0.59,2.00,3.25,4.41,1]$} & {$[2.29,2.70,2.70,3.21,0.42]$} & 2.59 & 2.59 & 0.93 & 0.0 \\
\hline$(12)$ & {$[0.38,2.50,5.00,7.83,1]$} & {$[2.88,3.61,3.61,4.21,0.35]$} & 3.90 & 3.94 & 0.93 & 0.5 \\
\hline$(13)$ & {$[1.17,3.50,5.50,7.83,1]$} & {$[4.09,4.65,4.65,5.41,0.40]$} & 4.56 & 4.57 & 0.95 & 0.1 \\
\hline$(14)$ & {$[2.59,4.00,5.50,7.62,1]$} & {$[4.29,4.75,4.75,5.21,0.38]$} & 4.95 & 4.98 & 0.89 & 0.6 \\
\hline$(15)$ & {$[2.17,4.25,6.00,7.83,1]$} & {$[4.79,5.29,5.29,6.02,0.41]$} & 5.13 & 5.13 & 0.98 & 0.0 \\
\hline$(16)$ & {$[3.59,4.75,5.50,6.91,1]$} & {$[4.86,5.03,5.03,5.14,0.27]$} & 5.19 & 5.21 & 0.90 & 0.6 \\
\hline$(17)$ & {$[3.59,4.75,6.00,7.41,1]$} & {$[4.79,5.30,5.30,5.71,0.42]$} & 5.41 & 5.41 & 0.99 & 0.0 \\
\hline$(18)$ & {$[3.38,5.50,7.50,9.62,1]$} & {$[5.79,6.50,6.50,7.21,0.41]$} & 6.50 & 6.50 & 0.82 & 0.0 \\
\hline$(19)$ & {$[4.38,6.50,8.00,9.41,1]$} & {$[6.79,7.38,7.38,8.21,0.49]$} & 7.16 & 7.15 & 0.82 & 0.2 \\
\hline$(20)$ & {$[4.38,6.50,8.00,9.41,1]$} & {$[6.79,7.38,7.38,8.21,0.49]$} & 7.16 & 7.15 & 0.90 & 0.2 \\
\hline$(21)$ & {$[4.38,6.50,8.25,9.62,1]$} & {$[7.19,7.58,7.58,8.21,0.37]$} & 7.25 & 7.21 & 0.86 & 0.7 \\
\hline$(22)$ & {$[5.38,7.50,8.75,9.81,1]$} & {$[7.79,8.22,8.22,8.81,0.45]$} & 7.90 & 7.87 & 0.86 & 0.6 \\
\hline$(23)$ & {$[5.38,7.50,8.75,9.83,1]$} & {$[7.69,8.19,8.19,8.81,0.47]$} & 7.91 & 7.88 & 0.45 & 0.6 \\
\hline$(24)$ & {$[5.38,7.50,8.75,9.81,1]$} & {$[7.79,8.30,8.30,9.21,0.53]$} & 8.01 & 8.01 & 0.65 & 0.0 \\
\hline$(25)$ & {$[5.38,7.50,9.00,9.81,1]$} & {$[8.29,8.56,8.56,9.21,0.38]$} & 8.03 & 7.97 & 0.90 & 1.3 \\
\hline$(26)$ & {$[5.98,7.75,8.60,9.52,1]$} & {$[8.03,8.36,8.36,9.17,0.57]$} & 8.12 & 8.12 & 0.65 & 0.0 \\
\hline$(27)$ & {$[7.37,9.41,10,10,1]$} & {$[8.72,9.91,10,10,1]$} & 9.30 & 9.31 & 0.24 & 0.3 \\
\hline$(28)$ & {$[7.37,9.82,10,10,1]$} & {$[9.74,9.98,10,10,1]$} & 9.31 & 9.23 & 0.28 & 3.0 \\
\hline$(29)$ & {$[7.37,9.59,10,10,1]$} & {$[8.95,9.93,10,10,1]$} & 9.34 & 9.35 & 0.28 & 0.3 \\
\hline$(30)$ & {$[7.37,9.73,10,10,1]$} & {$[9.34,9.95,10,10,1]$} & 9.37 & 9.34 & 0.47 & 1.1 \\
\hline$(31)$ & {$[7.37,9.82,10,10,1]$} & {$[9.37,9.95,10,10,1]$} & 9.38 & 9.34 & 0.48 & 1.5 \\
\hline$(32)$ & {$[8.68,9.91,10,10,1]$} & {$[9.61,9.97,10,10,1]$} & 9.69 & 9.67 & 0.36 & 1.5 \\
\hline
\end{tabular}

Difference (error) RMS\% compared to KM 0.95\%.

This method has a high degree of freedom (9), and the uncertainty removing method can be defined by a simple linear method or more complex methods based on conservative or courageous logic and on the conditions and requirements of different issues.

Our method eases the defuzzifying of IT2MF to obtain accurate results on the main feature of IT2FSs. In case of slight output differences between our method and the KM algorithm, no mathematic proof exists that shows that the outputs gained by KM algorithm are better than our method. On the contrary, the existence of concepts and reasons behind our proposed method provides a designer with more opportunities to manage parameters related to uncertainty in IT2 fuzzy controllers in engineering and industrial affairs conveniently.

\section{Simulations and Investigations}

Investigation 1 examines the affectivity of the proposed uncertainty measures. Investigation 2 shows the illustrative concept of the effect of uncertainty on membership degrees. Investigation 3 shows the comparative outputs as a result of applying the proposed formula and KM algorithm of 32 basic IT2MF words. A single-input single-output (SISO) fuzzy system is introduced in "Investigation 4" to compare clearly the outputs created in different uncertainty imbalance situations and by different methods. Investigation 5 discusses on a comparison between collapsing method [43] and proposed method. In Investigation 6, the effects of different types of uncertainty on system output are investigated. Examples are chosen by selecting various forms of MFs and changing 


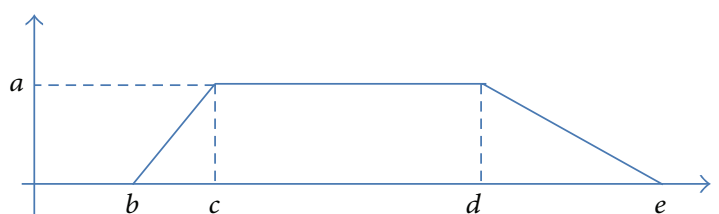

FIgURE 11: Common form of trapezoidal and triangle MFs.

the different modes of antecedent and subsequent MFs to create various modes by using different ARSs and membership degrees and cover the generalities of the issue. The root mean square of the differences and maximum error shows different states.

The common form of the trapezoidal and triangular MF can be described by a five-number vector $[a, b, c, d$, and e] (Figure 11). This presentation of the common form of trapezoidal and triangular MF is used in the rest of this paper. The distance between " $b$ " and " $e$," that is, $(e-b)$ is called the support of MF (Figure 11).

Investigation 1. Calculate $\alpha$ and $\beta$ for comparing uncertainty of two IT2MFs shown in Figure 12. For calculating $\beta$ consider $n=3$.

According to (4), the new domain $d$ which is necessary for calculating $\alpha$ and $\beta$ is calculable as follows:

$$
\begin{gathered}
\frac{1}{2} d^{2}-d+\left(\int_{0}^{1} 0.5 y d y-\int_{0}^{1} 0.5 d y-\frac{1}{2}+1\right)=0 \\
\Longrightarrow \frac{1}{2} d^{2}-d+\left(\frac{1}{4}-\frac{1}{2}-\frac{1}{2}+1\right)=0 \\
\Longrightarrow \frac{1}{2} d^{2}-d+\frac{1}{4}=0 \Longrightarrow d=1+\frac{\sqrt{2}}{2}
\end{gathered}
$$

Calculating $\alpha$. COG1 and COG2 based on KM algorithm are 1.01 and 1.1, respectively, then $\alpha 1$ and $\alpha 2$ are 0.01 and 0.1 , respectively.

Two uncertainty measures have been shown in Figure 13. Considering the area of surrounded region by upper and lower bounds, it is clear that the uncertainty of Figure 12(a) is lesser than the uncertainty of Figure 12(b) before calculation. This fact is confirmed using the proposed method for uncertainty measurement. Calculations for $\beta$ using (8) show $\beta_{1}=0.003$ and $\beta_{2}=0.02$. A comparison between $\alpha$ and $\beta$ shows that the sensitivity of $\alpha$ is more than $\beta$.

Investigation 2. For IT2MF (UMF $=[0.38,2.50,3.75,4.81,1]$ and LMF $=[2.79,3.30,3.30,4.21,0.53])$ calculate (a) ARS and (b) output value by using (10). (c) Calculate the difference percentage between obtained value by using (12) and the COG of IT2MF.

Answer. The COG of the principal function shown in Figure $14(\mathrm{a})$ is $M=2.88$. In Figure 14(b), the function of $\underline{u}=\mu_{u}-\mu_{l}$ is observed, wherein, $A_{l}=7.15, A_{r}=5.16$, and ARS $=0.72$ are obtained from (2).
By using (10), the embedded fuzzy $\mathrm{T} 1$ shown in Figure 14(a) is obtained. The COG of this fuzzy T1 is easily calculable as COG $(\mathrm{D} 3)=3.0088$.

The COG of IT2MF in Figure 14(a) (i.e., 3.01) is obtained by using the KM algorithm. To calculate the difference percentage between these two COGs, we use the following formula:

$$
E=\frac{\mid \text { Output }(10)-\mathrm{COG}(\mathrm{IT} 2 \mathrm{MF}) \mid}{\operatorname{Sup}(\mathrm{UMF})} \times 100 \%,
$$

where Sup is the support of MF (Figure 11).

Here, $E=0.04 \%$, and (10) produces accurate answers to COG (IT2MF).

Investigation 3. Wu and Mendel showed in [18] the defuzzified output of 32 basic IT2 fuzzy words based on the KM algorithm. Here, we calculate the defuzzified output by using our proposed formula (i.e., (10) for obtaining T1MF and using COG for type-1 defuzzification). Table 2 shows those words by using the five parameters for introducing trapezoidal MFs shown in Figure 11. Equation (12) is used for calculating $(E)$, which shows the error percentages of (10). The absolute maximum error of (10) for those words is $3 \%$.

Investigation 4. A SISO single-rule system exists. The specifications of this system are as follows.

Rule. If $X$ is small, then $Y$ is small. Implication $=$ Max.

$Y$ (output) MF is T1: small $=\left[\begin{array}{lllll}0 & 0 & 0 & 2.95 & 1\end{array}\right]$.

$X$ (input) $M F$ is the (a) T1MF shown in Figure 4(b); (b) standard IT2MF shown in Figure 5(a); (c) IT2MF shown in Figure 4(c); (d) IT2MF shown in Figure 5(c).

Calculate the output by using (9) and investigate the effect of uncertainty.

The output is affected by various uncertainties separately (Figure 15). For example, we want to discuss the effect of data uncertainty only. Considering the IT2Mf of $x$, a lower " $x$ " $(x<2.5)$ is more uncertaintified (compared with the conditions in which uncertainties are absent). A higher " $x$ " $(7>x>2.5)$ is more uncertaintified, and $x>7$ indicates balance. If we look to the property of the rule used in this system and consider the bandwidth of uncertainty in input MF, higher values of " $y$ " are more certain if $x<2$, and lower values of " $y$ " are more certain if $7>x>2.5$; in other conditions, " $y$ " does not change. This reasoning is applicable for other types of uncertainty.

Investigation 5. Calculate outputs by using collapsing method [43] and (9) for

(a) the MF of the upper and lower bands that are 0.8 and 0.2 in the [ $\left.\begin{array}{ll}0 & 1\end{array}\right]$ range of the primary domain;

(b) symmetric triangle MF, UMF $=\left[\begin{array}{lllll}0.1 & 0.4 & 0.4 & 0.7 & 1\end{array}\right]$ and $\mathrm{LMF}=\left[\begin{array}{lllll}0.2 & 0.4 & 0.4 & 0.6 & 1\end{array}\right]$; 


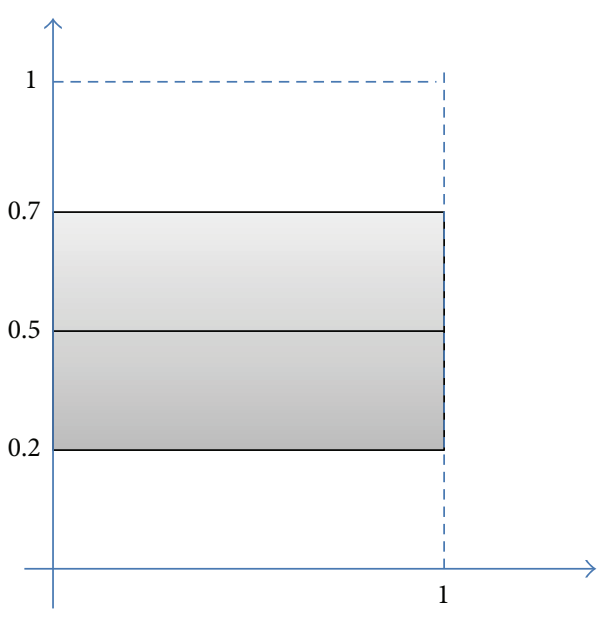

(a)

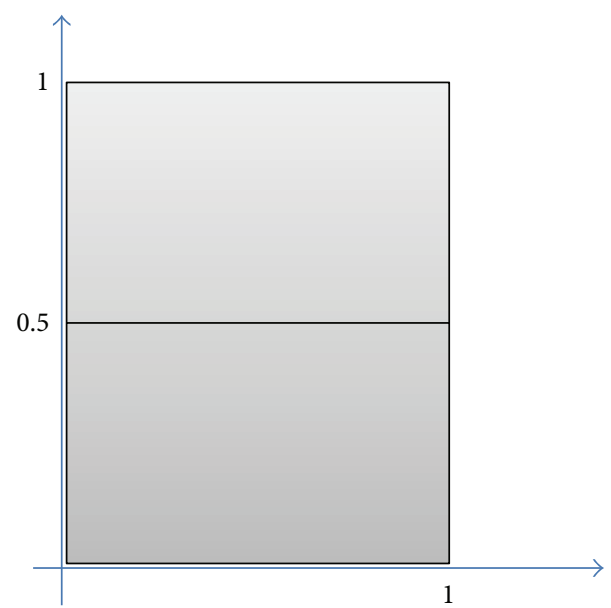

(b)

FIGURE 12: Two IT2MFs with equal domains and different uncertainty bands.

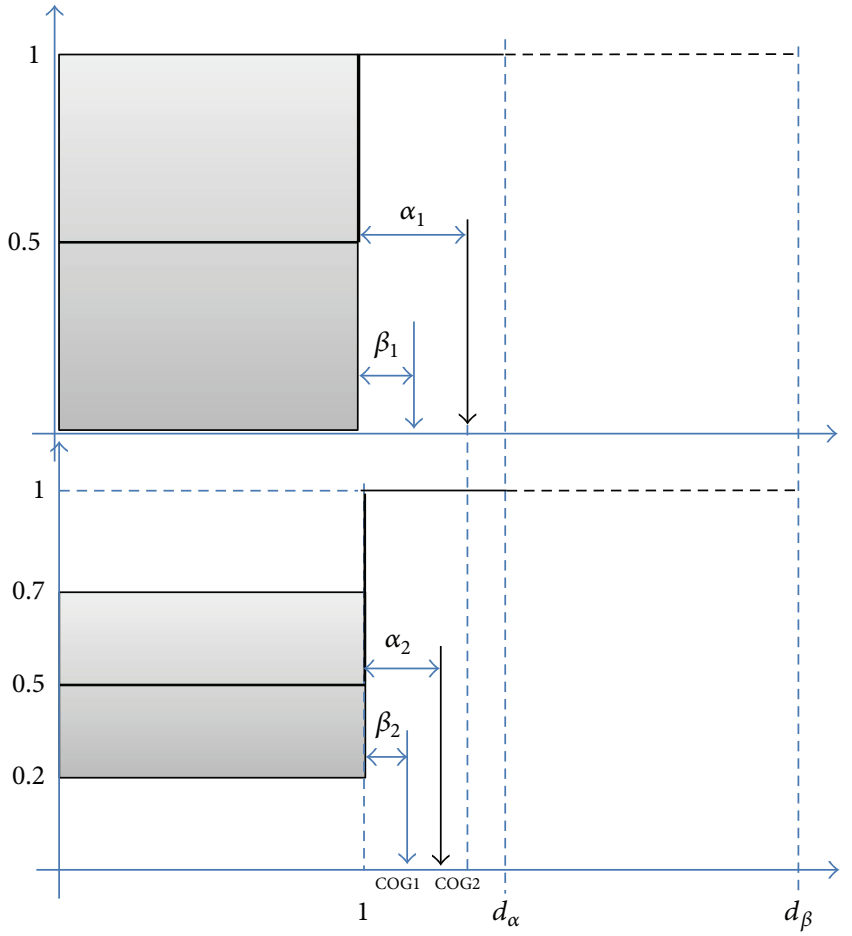

FIGURE 13: COG of extended IT2MF and position of $\alpha$ and $\beta, d_{\beta}=$ $3 d_{\alpha}$.

(c) asymmetric Gaussian MF, UMF $=\left[\begin{array}{ll}0.3 & 0.2\end{array}\right]$ and LMF $=\left[\begin{array}{ll}0.18 & 0.3\end{array}\right]$.

Answer. In case of symmetric IT2MFs, the use of (9) causes no error. The error is zero when the upper and lower bands are considered continuous functions or applied with few samples. The defuzzified outputs for the upper and lower bands are 0.5 and 0.4 , respectively. The collapsing method with 11 or more samples is slightly different with the exact answer. For the asymmetric Gaussian IT2MF, the result obtained by (10) for 100 samples is 0.33 ; the result decreases with increasing the number of samples. By using continuous formula of the upper and lower MFs, the output converges to 0.3219 . The results in [43] are slightly different with the convergent answer.

Investigation 6. Provide visualization on the effect of different types of uncertainty by investigating the output of an SISO FLC. The SISO inputs are selected from Section 2 of this paper.

Rule. If $X$ is small, then $Y$ is small. Implication $=$ Max.

$$
Y \text { (output) MF is T1: small }=\left[\begin{array}{lllll}
0 & 0 & 0 & 4.95 & 1
\end{array}\right] .
$$

$X$ (input) $M F$ is the (a) T1MF shown in Figure 4(b); the standard IT2MF shown in Figure 5(a); (c) IT2MF shown in Figure 4(c); (d) IT2MF shown in Figure 5(c).

In all the above conditions, calculate the output by using (9), and investigate the effect of uncertainty.

The output is affected by various uncertainties separately (Figure 16). Figures 16(a), 16(b), and 16(c) show that applied uncertainty causes rules to weaken. We use the following rule for this case: "if the input is small, the output is small."

However, we considered the effect of uncertainty in Figure 16. We observed in all cases that the response after applying the uncertainty increased, as well as in points in which input data has greater amount of uncertainty. Output also significantly increased. The result shows that the concept of "low" in the output shifts toward the concept of "large."

Discussion on Investigations. The proposed method has a high degree of freedom. The uncertainty removing method can be defined by a simple linear method or more complex methods based on conservative or courageous logic and the conditions and requirements of different issues.

Our method eased the defuzzifying of IT2MF to obtain accurate results on the feature of IT2FSs. In case of slight 


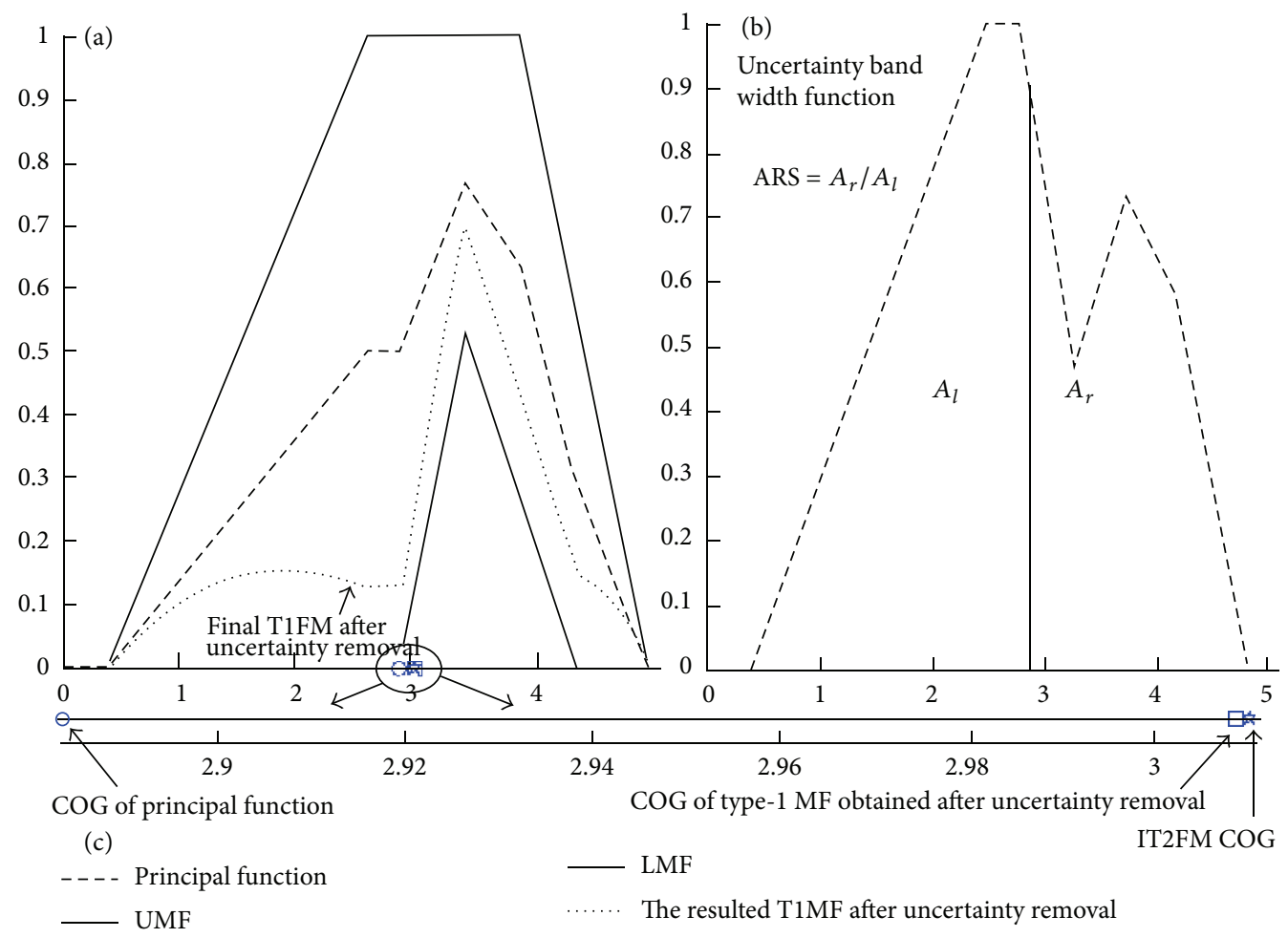

FIGURE 14: (a) IT2MF and its principal and output-based imbalanced uncertainty; (b) uncertain bandwidth function; (c) COGs of principal, output-based uncertainty imbalances, and IT2MF (KM) functions.

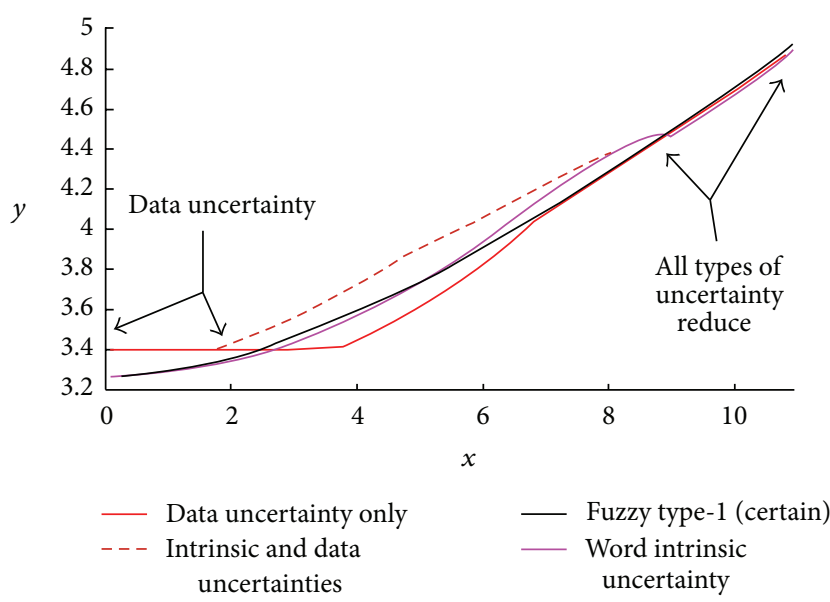

FIGURE 15: Effect of uncertainty on outputs of SISO (Investigation $4)$.

output differences between our method and the KM algorithm, no mathematical proof exists to show that the outputs gained by KM algorithm are better than our method. On the contrary, the existence of concepts and reasons behind our proposed method provides designers with more opportunities to manage parameters related to uncertainty in T2 fuzzy systems in engineering and industrial affairs conveniently.

Designers who work with fuzzy systems must have information on the behavior of the fuzzifier, inference, and defuzzifier methods used in the system. No defuzzification method exists that is suitable in all systems and all conditions.
If method $\mathrm{A}$ produces better results than method $\mathrm{B}, \mathrm{B}$ is better than $\mathrm{A}$ if conditions or systems change. The experience of a designer plays a major role in the selection of an appropriate method. In our case, understanding and absorbing system behavior is easier.

\section{Conclusion}

This paper presented the Theorem of Uncertainty Avoidance and used it for uncertainty measuring in IT2FMs. The proposed methods provide simple closed formulas for calculating total uncertainty of a membership function. This paper brought up a new vision to the problem of uncertainty measure. The measurement is based on the power of uncertainty to push the COG of principal function to a completely certain domain. The results of this paper provide a new perspective on the relationship between uncertainty and fuzzy system output. For each T1MF, uncertaintified functions are presented to be more complete than common IT2MFs. In addition, we show that uncertainty reduces the value of membership degrees and the absolute value of words. Higher uncertainty causes a higher reduction of values. For example, if the input and output of a system contain "low," "medium," and "large" words, after injecting uncertainty to inputs, "low" will shift to "medium," "medium" to "large," and "large" to "medium." Results show that the uncertainty reduces the value of the membership degree proportionally. The concept of words is also shifted toward the opposite neighbor words in the system output by the uncertainty of 


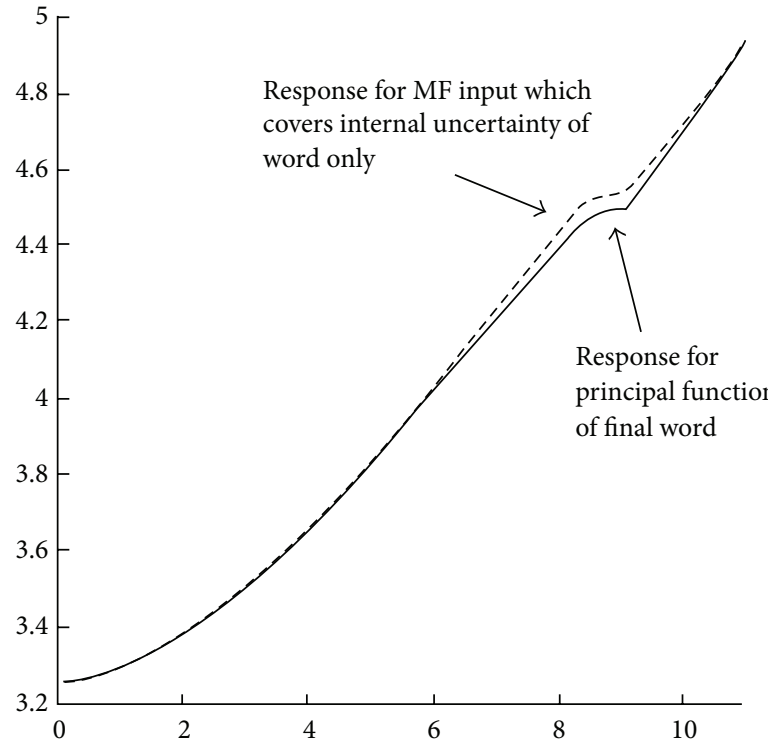

(a)

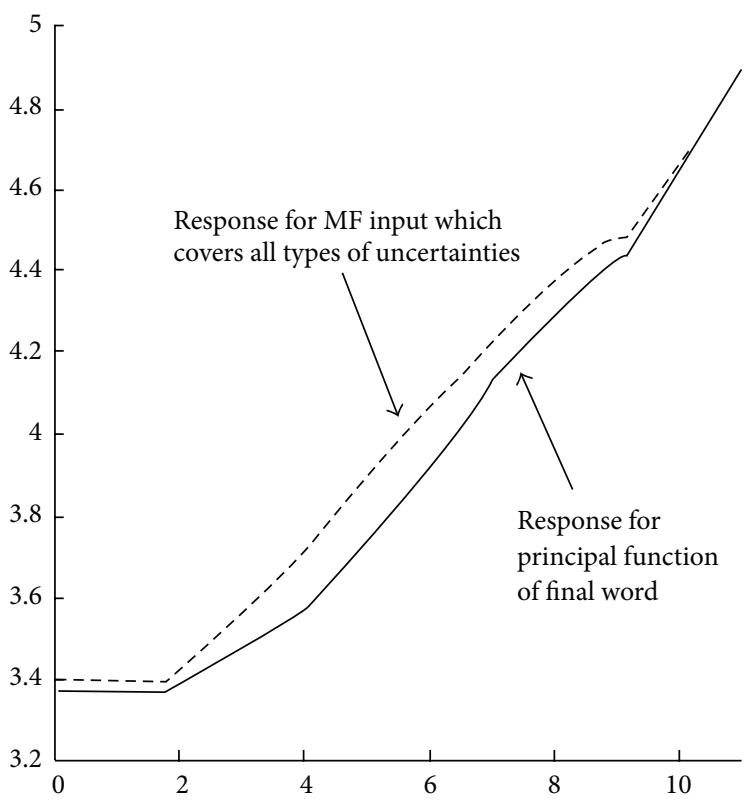

(c)

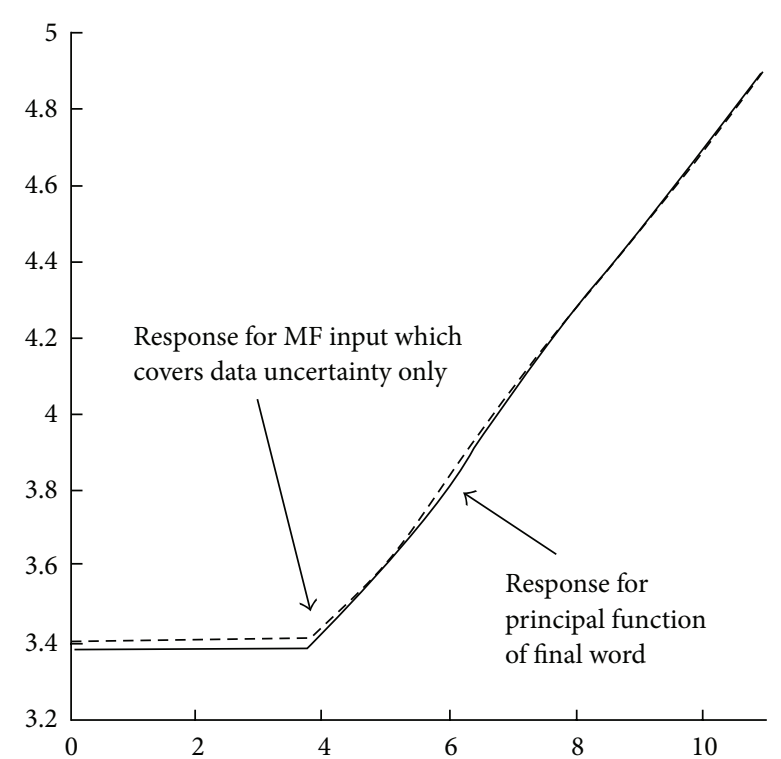

(b)

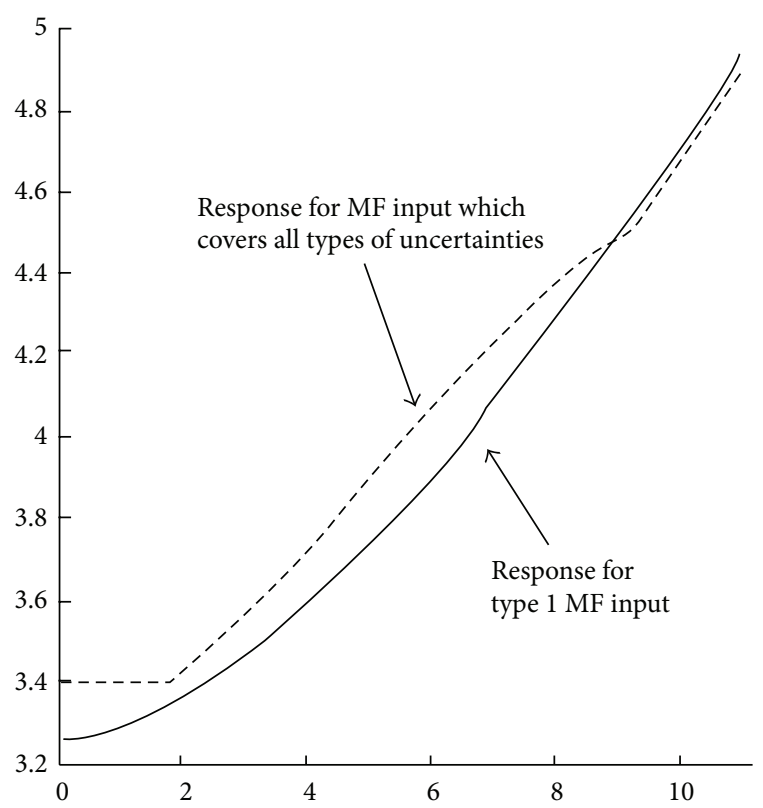

(d)

FIGURE 16: Effect of uncertainty on outputs of a simple fuzzy controller (Investigation 6).

system inputs proportionally. The proposed technique for uncertainty removing can be considered as a closed formula for calculating the COG of IT2MF with acceptable accuracy. On the contrary, the existence of concepts and reasons behind the new interpretation of uncertainty provides designers more opportunities to manage parameters related to uncertainty in interval T2 fuzzy controllers in engineering and industrial affairs conveniently.

\section{Acknowledgment}

The authors would like to acknowledge the support of the Universiti Sains Malaysia fellowship.

\section{References}

[1] L. A. Zadeh, "The concept of a linguistic variable and its application to approximate reasoning-I," Information Sciences, vol. 8, no. 3, pp. 199-249, 1975.

[2] L. A. Zadeh, "The concept of a linguistic variable and its application to approximate reasoning-II," Information Sciences, vol. 8, no. 4, pp. 301-357, 1975.

[3] H. Hagras, "Type-2 FLCs: a new generation of fuzzy controllers," IEEE Computational Intelligence Magazine, vol. 2, no. 1, pp. 30-43, 2007.

[4] R. John and S. Coupland, "Type-2 fuzzy logic: a historical view," IEEE Computational Intelligence Magazine, vol. 2, no. 1, pp. 57$62,2007$. 
[5] F. Liu and J. M. Mendel, "Aggregation using the fuzzy weighted average as computed by the Karnik-Mendel algorithms," IEEE Transactions on Fuzzy Systems, vol. 16, no. 1, pp. 1-12, 2008.

[6] F. Liu and J. M. Mendel, "Encoding words into interval type2 fuzzy sets using an interval approach," IEEE Transactions on Fuzzy Systems, vol. 16, no. 6, pp. 1503-1521, 2008.

[7] M. Melgarejo and C. A. Pena-Reyes, "Implementing interval type-2 fuzzy processors," IEEE Computational Intelligence Magazine, vol. 2, no. 1, pp. 63-71, 2007.

[8] J. M. Mendel, Uncertain Rule-Based Fuzzy Logic Systems: Introduction and New Directions, Prentice-Hall, Upper-Saddle River, NJ, USA, 2001.

[9] J. M. Mendel, "Type-2 fuzzy sets and systems: an overview," IEEE Computational Intelligence Magazine, vol. 2, no. 1, pp. 2029, 2007.

[10] J. M. Mendel, "Advances in type-2 fuzzy sets and systems," Information Sciences, vol. 177, no. 1, pp. 84-110, 2007.

[11] J. M. Mendel, "Computing with words: zadeh, turing, popper and occam," IEEE Computational Intelligence Magazine, vol. 2, no. 4, pp. 10-17, 2007.

[12] J. M. Mendel, R. I. John, and F. Liu, "Interval type-2 fuzzy logic systems made simple," IEEE Transactions on Fuzzy Systems, vol. 14, no. 6, pp. 808-821, 2006.

[13] J. M. Mendel, H. Hagras, and R. I. John, "Standard background material about interval type-2 fuzzy logic systems that can be used by all authors," http://ieee-cis.org/_ files/standards.t2.win.pdf.

[14] D. Wu and J. M. Mendel, "Uncertainty measures for interval type-2 fuzzy sets," Information Sciences, vol. 177, no. 23, pp. 5378-5393, 2007.

[15] D. Wu and J. M. Mendel, "Aggregation using the linguistic weighted average and interval type-2 fuzzy sets," IEEE Transactions on Fuzzy Systems, vol. 15, no. 6, pp. 1145-1161, 2007.

[16] J. M. Mendel and D. Wu, "Cardinality, fuzziness, variance and skewness of interval type-2 fuzzy sets," in Proceedings of the IEEE Symposium on Foundations of Computational Intelligence (FOCI '07), pp. 375-382, April 2007.

[17] J. M. Mendel, "Computing with words: zadeh, turing, popper and occam," IEEE Computational Intelligence Magazine, vol. 2, no. 4, pp. 10-17, 2007.

[18] D. Wu and J. M. Mendel, "A comparative study of ranking methods, similarity measures and uncertainty measures for interval type-2 fuzzy sets," Information Sciences, vol. 179, no. 8, pp. 1169-1192, 2009.

[19] R. John, "Type 2 fuzzy sets: an appraisal of theory and applications," International Journal of Uncertainty, Fuzziness and Knowlege-Based Systems, vol. 6, no. 6, pp. 563-576, 1998.

[20] G. J. Klir and B. Parviz, "Probability-possibility transformations: a comparison," International Journal of General Systems, vol. 21, no. 3, pp. 291-310, 1992.

[21] S. Aminifar and A. bin Marzuki, "Horizontal and vertical rule bases method in fuzzy controllers," Mathematical Problems in Engineering, vol. 2013, Article ID 532046, 9 pages, 2013.

[22] L. A. Zadeh, "Toward a generalized theory of uncertainty (GTU)- An outline," Information Sciences, vol. 172, no. 1-2, pp. 1-40, 2005.

[23] G. J. Klir, "Principles of uncertainty: what are they? Why do we need them?" Fuzzy Sets and Systems, vol. 74, no. 1, pp. 15-31, 1995.
[24] G. J. Klir and T. A. Folger, Fuzzy Sets, Uncertainty, and Information, Prentice-Hall, Englewood Cliffs, NJ, USA, 1988.

[25] D. Harmanec, "Measures of uncertainty and information, Society for Imprecise Probability Theory and Applications," 1999, http://www.sipta.org/documentation/summary_measures/ main.html.

[26] R. Christensen, "Entropy minimax multivariate statistical modeling I: theory," International Journal of General Systems, vol. 11, no. 3, pp. 231-277, 1985.

[27] R. Christensen, "Entropy minimax multivariate statistical modeling II: applications," International Journal of General Systems, vol. 12, no. 3, pp. 227-305, 1985.

[28] G. J. Klir, "A principle of uncertainty and information invariance," International Journal of General Systems, vol. 17, no. 2-3, pp. 249-275, 1990.

[29] G. J. Klir and B. Parviz, "Probability-possibility transformations: a comparison," International Journal of General Systems, vol. 21, no. 3, pp. 291-310, 1992.

[30] L. A. Zadeh, "Toward a theory of fuzzy information granulation and its centrality in human reasoning and fuzzy logic," Fuzzy Sets and Systems, vol. 90, no. 2, pp. 111-127, 1997.

[31] L. A. Zadeh, "Fuzzy sets and information granularity," in Advances in Fuzzy Set Theory and Applications, M. Gupta, R. Ragade, and R. Yager, Eds., pp. 3-18, North-Holland Publishing, Amsterdam, The Netherlands, 1979.

[32] M. Higashi and G. Klir, "Measures of uncertainty and information based on possibility distributions," in Fuzzy Sets For Intelligent Systems, pp. 217-232, Morgan Kaufman Publishers, San Mateo, Calif, USA, 1993.

[33] J. M. Mendel, "Fuzzy sets for words: a new beginning," in Proceedings of the IEEE International conference on Fuzzy Systems, pp. 37-42, St. Louis, Mo, USA, May 2003.

[34] N. N. Karnik and J. M. Mendel, "Centroid of a type-2 fuzzy set," Information Sciences, vol. 132, no. 1-4, pp. 195-220, 2001.

[35] J. M. Mendel and R. I. B. John, “Type-2 fuzzy sets made simple," IEEE Transactions on Fuzzy Systems, vol. 10, no. 2, pp. 117-127, 2002.

[36] O. Linda and M. Manic, "Uncertainty modeling for interval type-2 fuzzy logic systems based on sensor characteristics," in Proceedings of the IEEE Symposium onAdvances in Type-2 Fuzzy Logic Systems (T2FUZZ '11), pp. 31-37, April 2011.

[37] B. Choi and F. Chung-Hoon Rhee, "Interval type-2 fuzzy membership function generation methods for pattern recognition," Information Sciences, vol. 179, no. 13, pp. 2102-2122, 2009.

[38] J. M. Mendel, "Computing with words and its relationships with fuzzistics," Information Sciences, vol. 177, no. 4, pp. 988-1006, 2007.

[39] $\mathrm{D}$. Wu, "On the fundamental differences between interval type2 and type-1 fuzzy logic controllers in common systems," IEEE Transactions on Fuzzy Systems, vol. 20, no. 5, pp. 832-848, 2012.

[40] W. L. Oberkampf, S. M. DeLand, B. M. Rutherford, K. V. Diegert, and K. F. Alvin, "Error and uncertainty in modeling and simulation," Reliability Engineering and System Safety, vol. 75, no. 3, pp. 333-357, 2002.

[41] L. J. Lucas, H. Owhadi, and M. Ortiz, "Rigorous verification, validation, uncertainty quantification and certification through concentration-of-measure inequalities," Computer Methods in Applied Mechanics and Engineering, vol. 197, no. 51-52, pp. 45914609, 2008. 
[42] C. J. Roy and W. L. Oberkampf, "A comprehensive framework for verification, validation, and uncertainty quantification in scientific computing," Computer Methods in Applied Mechanics and Engineering, vol. 200, no. 25-28, pp. 2131-2144, 2011.

[43] S. Greenfield, F. Chiclana, S. Coupland, and R. John, "The collapsing method of defuzzification for discretised interval type-2 fuzzy sets," Information Sciences, vol. 179, no. 13, pp. 2055-2069, 2009. 


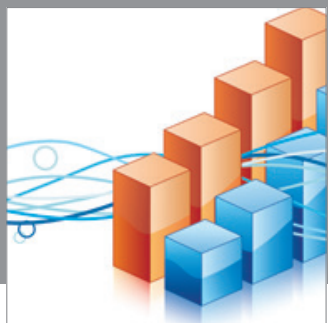

Advances in

Operations Research

mansans

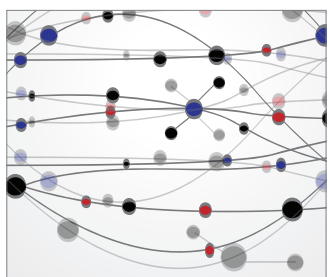

The Scientific World Journal
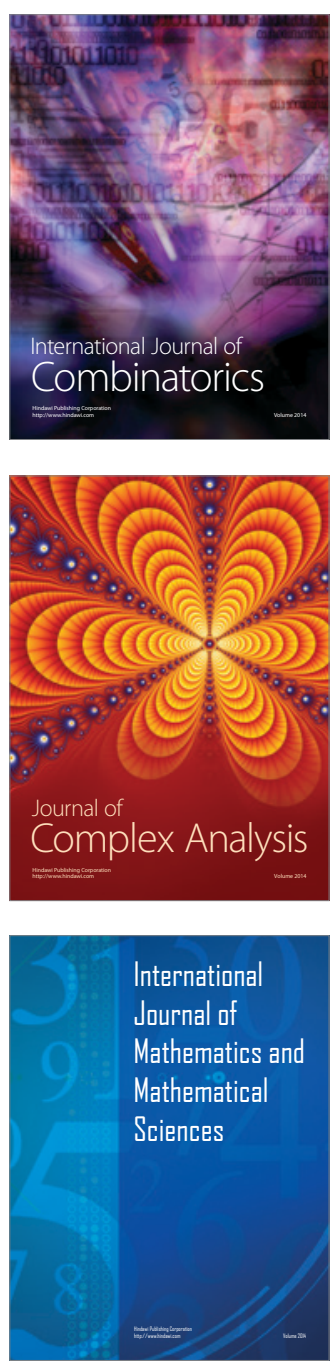
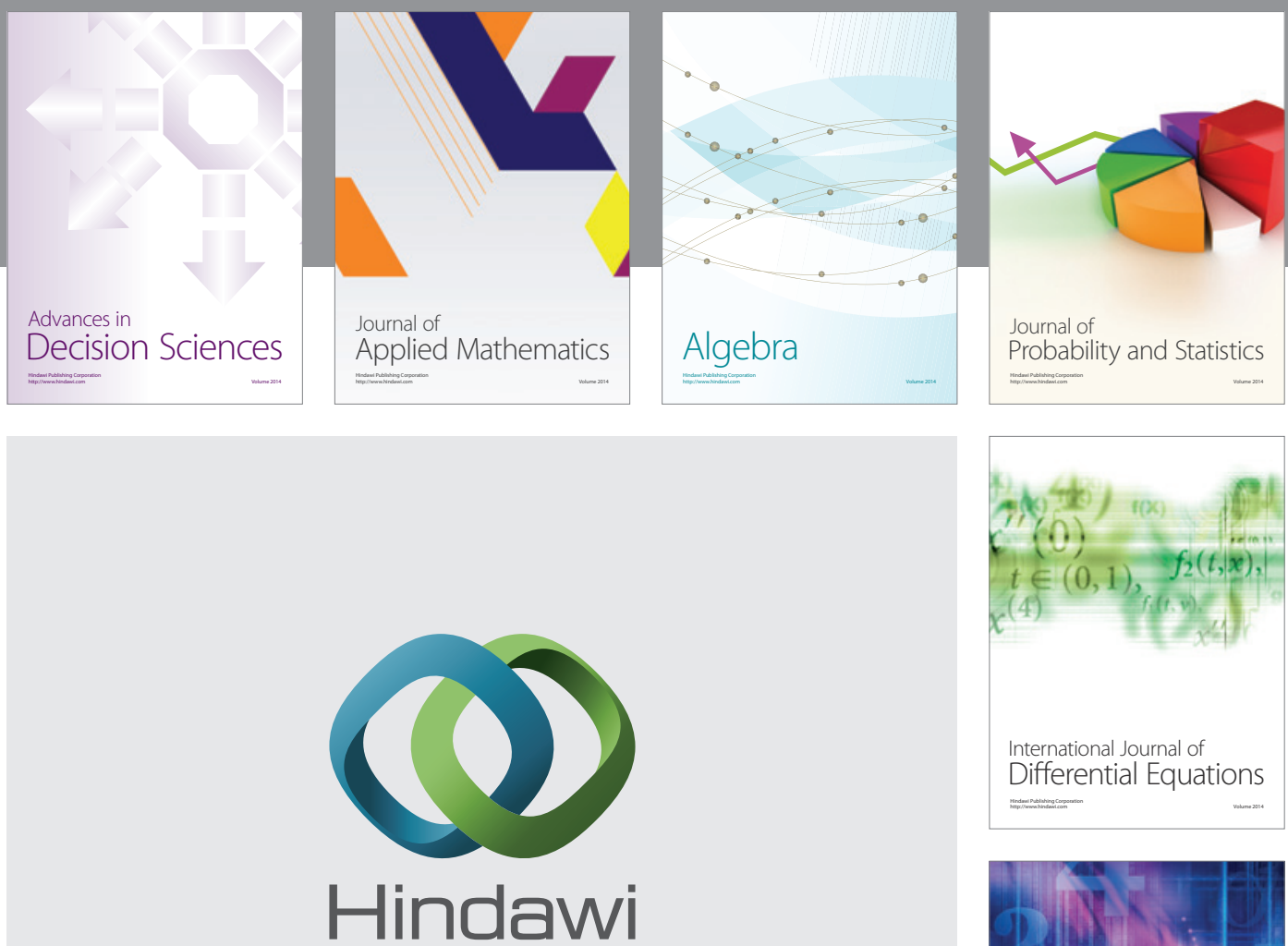

Submit your manuscripts at http://www.hindawi.com
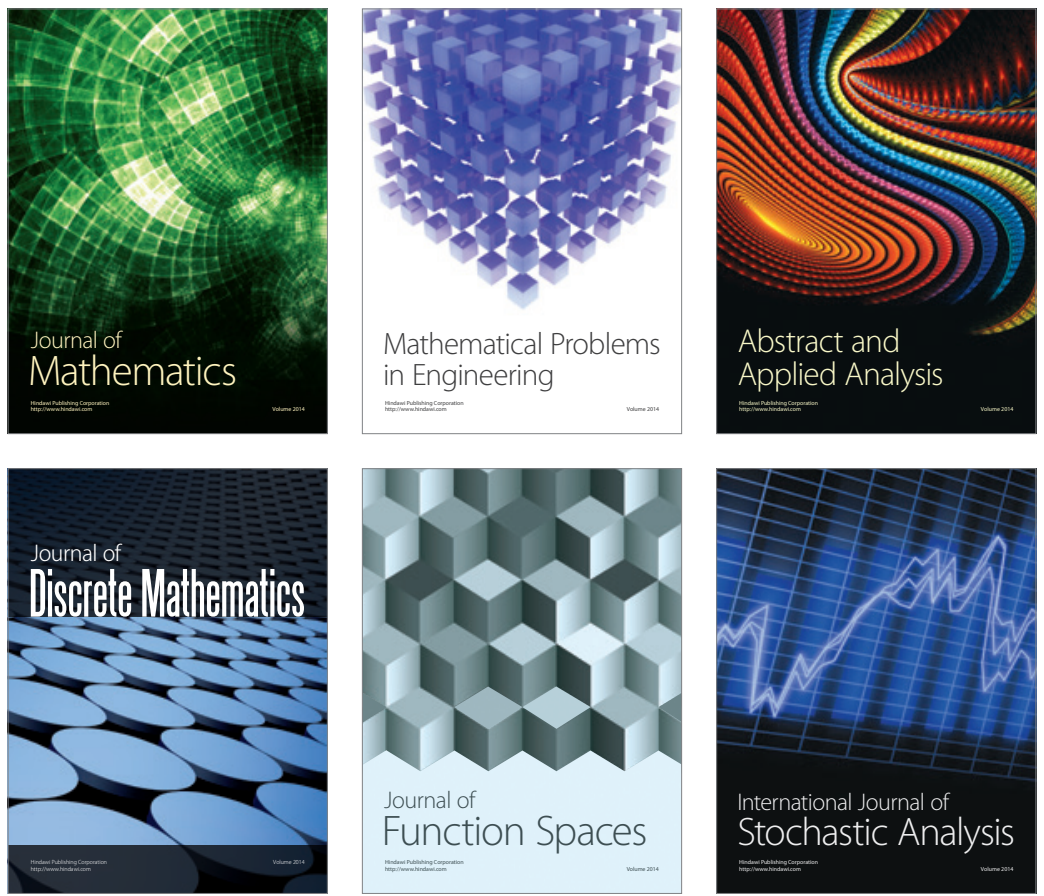

Journal of

Function Spaces

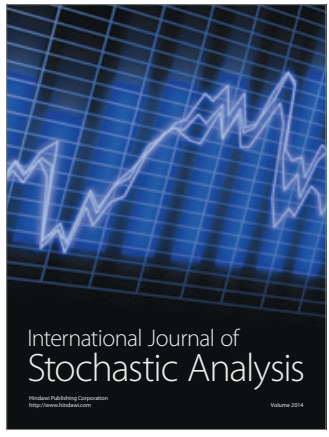

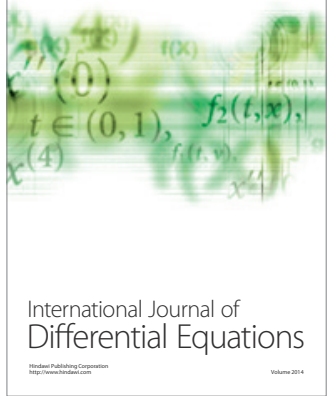
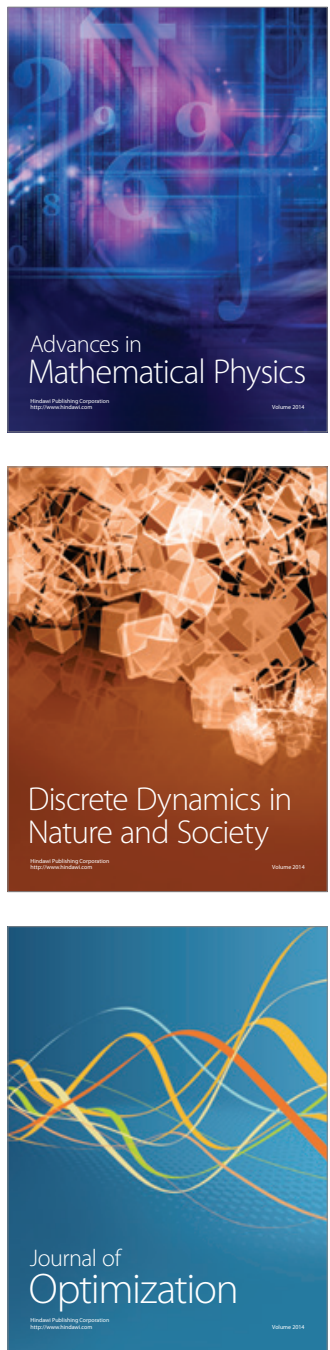